\title{
Tulane
}

\author{
Tulane Economics Working Paper Series
}

\section{Threshold Uncertainty in the Private-Information Subscription Game}

\author{
Stefano Barbieri \\ Department of Economics \\ 206 Tilton Hall \\ Tulane University \\ New Orleans, LA 70118 \\ sbarbier@tulane.edu
}

\author{
David A. Malueg \\ Department of Economics \\ 3136 Sproul Hall \\ UC Riverside \\ Riverside, CA 92521-0427 \\ david.malueg@ucr.edu
}

Working Paper 0903

February 2009

\begin{abstract}
We introduce threshold uncertainty, à la Nitzan and Romano (1990), into a private-values model of voluntary provision of a discrete public good. Players are allowed to make any level of contribution toward funding the good, which is provided only if the cost threshold is reached. Otherwise, contributions are refunded. Conditions ensuring existence and uniqueness of a Bayesian equilibrium are established. Further restricting the threshold uncertainty to a uniform distribution, we show the equilibrium strategies are very simple, even allowing for any number of players with asymmetric distributions of values. Comparative statics with respect to changes in players' distributions are derived, allowing changes in both the intensity and the dispersion of values. Finally, we show the equilibrium is interim incentive inefficient. The sharpness of our results greatly contrasts with the more qualified insights of earlier private-values models with known cost threshold, which relied on there being two symmetric players and generally exhibited multiple equilibria.
\end{abstract}

Keywords: discrete public good, subscription game, threshold uncertainty JEL codes: H41, D61, D82 


\title{
Threshold uncertainty in the private-information subscription game
}

\author{
Stefano Barbieri* \\ David A. Malueg ${ }^{\dagger}$
}

February 3, 2009

\begin{abstract}
We introduce threshold uncertainty, à la Nitzan and Romano (1990), into a private-values model of voluntary provision of a discrete public good. Players are allowed to make any level of contribution toward funding the good, which is provided only if the cost threshold is reached. Otherwise, contributions are refunded. Conditions ensuring existence and uniqueness of a Bayesian equilibrium are established. Further restricting the threshold uncertainty to a uniform distribution, we show the equilibrium strategies are very simple, even allowing for any number of players with asymmetric distributions of values. Comparative statics with respect to changes in players' distributions are derived, allowing changes in both the intensity and the dispersion of values. Finally, we show the equilibrium is interim incentive inefficient. The sharpness of our results greatly contrasts with the more qualified insights of earlier private-values models with known cost threshold, which relied on there being two symmetric players and generally exhibited multiple equilibria.
\end{abstract}

JEL Codes: H41, D61, D82

Keywords: discrete public good, subscription game, threshold uncertainty

624

\section{DRAFT: Comments welcome}

*Department of Economics, 206 Tilton Hall, Tulane University, New Orleans, LA 70118; email: sbarbier@tulane.edu.

${ }^{\dagger}$ Department of Economics, 3136 Sproul Hall, University of California, Riverside, CA 92521; email: david.malueg@ucr.edu. 


\section{Introduction}

We analyze the effects of introducing threshold uncertainty, à la Nitzan and Romano (1990), into the privatevalues subscription game for provision of a discrete public good. A discrete (sometimes also called a binary or threshold) public good is either provided or not-quantity is not otherwise variable. The subscription game is a voluntary provision mechanism for such goods: Individuals privately contribute money; if total contributions reach a cost threshold, that is, if they suffice to fund the good, then provision occurs. Otherwise, contributions are refunded. The subscription game, along with the closely related contribution game (where contributions are not refunded), has been used extensively to describe situations including fund drives for public radio, contributions to neighborhood security programs, and capital campaigns for new university buildings. Beyond such standard examples of private provision of public goods, insights from the study of the private provision of a discrete public good have proven useful in the analysis of many issues, including bargaining (e.g. Mailath and Postlewaite, 1990) and common agency (e.g. Martimort and Moreira, 2007).

The full-information subscription game is well-understood (see Palfrey and Rosenthal, 1984, and Admati and Perry, 1991). In contrast, situations in which agents' values are private information have proven more difficult to analyze, especially when a mechanism designer with commitment power does not exist. Indeed, as Martimort and Moreira (2007) write, “... the provision of public goods under asymmetric information has mostly been viewed as a mechanism design problem under the aegis of an uninformed mediator having a full commitment ability" (p. 1). But, as they point out, “... in much real-world settings, centralized mechanisms and uninformed mediators with a strong ability to commit to those mechanisms might not be available" (p. 2$){ }^{1}$

Our paper belongs to the strand of the literature assuming that either such a mediator does not exist, or, if one does, then it has no commitment power. Focusing in particular on the known-threshold subscription game with private values and continuous contribution strategies, this literature includes Alboth et al. (2001), Menezes et al. (2001), Laussel and Palfrey (2003), and Barbieri and Malueg (2008), along with Martimort and Moreira (2007). While these papers provide interesting insights, technical issues have limited the scope of their analysis in important ways:

1. Existence and characterization results are limited to the case of two players having independently and identically distributed values.

2. Description of equilibria often requires further assumptions about specific functional forms for the distribution of players' values.

\footnotetext{
${ }^{1}$ They provide examples including health, environment, global warming, terrorism, multilateral foreign aid, and lobbying.
} 
3. A pervasive non-uniqueness of equilibria hinders comparative statics analysis and muddles efficiency considerations.

The main difference between our model and the previous literature is that we do not consider a fixed threshold. Rather, we assume players are unsure about the exact cost of provision. Uncertainty in the threshold cost appears in Nitzan and Romano (1990) and McBride (2006). Both papers, in contrast to ours, assume players' values are common knowledge. Nitzan and Romano (1990) argue that uncertainty in the threshold cost may be a better assumption to capture real-world situations. We find that, beyond being more realistic for many actual economic situations, a theoretical upshot of the threshold uncertainty assumption turns out to be a large gain in the tractability of the subscription game equilibria.

Our first contribution is to overcome the three limitations in the literature outlined above. For the two-player case, we derive existence, uniqueness, and characterization of equilibria only assuming standard restrictions on the form of the threshold uncertainty, while allowing for asymmetry in players' values. For a uniformly distributed threshold, these results extend to any number of contributors. In addition, our characterization is very simple and equilibrium is tractable. One of the most interesting results of Nitzan and Romano (1990) is that threshold uncertainty qualitatively alters the properties of full-information subscription game equilibria, especially with regards to efficiency. Our first contribution shows that, for private value environments, this qualitative difference is larger in scope and sharper.

Our second contribution is more applied. Tractability and uniqueness of equilibrium make our environment useful for understanding design features of the subscription game. For example, it has been suggested that, compared to binary contribution possibilities (as in Palfrey and Rosenthal, 1984, for example), allowing for continuous contribution choices may yield greater contributions, and hence greater likelihood of provision. We develop an example showing that the equilibrium with continuous strategies yields contributions at least as large as when contributors are restricted to contribute either 0 or $b$, say; but for a carefully chosen value of $b$, the discrete-contribution model has the same expected contributions and probability of provision.

We then show how changes in both intensity and dispersion of one player's values affect the contribution behavior of all other players and we determine the overall effect on total contributions. First, we show that if a player's values increase, in the sense of first-order stochastic dominance, then that player's contributions increase and the others' decrease - but overall expected contributions increase and so, too, does the probability of provision. Second, if only one player's distribution of values becomes more uncertain, we establish not only that total contributions increase, but that the increase is entirely due to the player in question contributing more, while everyone else contributes less. Correspondingly, the payoff of the "more uncertain" player decreases, while the payoff of every other player increases. We relate these results to the empirical 
literature on lobbying surveyed in Potters and Sloof (1996) and to the papers analyzing advantages and disadvantages of heterogeneity and fragmentation in cooperative endeavors, a literature surveyed in Alesina and La Ferrara (2005). Moreover, our results complement McBride's (2006). While McBride (2006) focuses on the effects of changing the distribution of the threshold cost, we focus on the effects of changing the distributions of players' values. One of McBride's most interesting results is that increased uncertainty in the distribution of the threshold may increase equilibrium contributions, ${ }^{2}$ thus contradicting the usual intuition that inefficiency worsens as the uncertainty increases. Again, in our private-information setting, the results are sharper and richer: increased uncertainty in any agent's value will increases total equilibrium contributions, and, under symmetry, expected welfare unequivocally improves.

Our third and final contribution is to examine the efficiency properties of equilibrium in relation to the extant results for the subscription game with known threshold. In particular, Laussel and Palfrey (2003) show the subscription game may be interim incentive efficient, although they establish this only for a uniform distribution of values and for a range of cost parameters. Moreover, inefficient equilibria always exist. Martimort and Moreira (2007) confirm these efficiency results and extend them to distributions with linear hazard rate, but add "Perturbing even slightly the model and considering distributions which no longer have linear hazard rates makes checking (36) [the analytical condition for efficiency] a complex numerical exercise. We conjecture that, beyond those cutting-edge cases, interim efficiency never holds" (p. 17). Such mixed results and difficulties do not arise in our model: we show, when the threshold is uniformly distributed, that equilibrium is interim incentive inefficient for all distributions of values and any number of players.

The rest of the paper is organized as follows. Section 2 describes the model, presents a general existence result, and proves uniqueness of equilibrium for the two-player case. Section 3 considers the case of a uniformly distributed threshold and characterizes the unique equilibrium for any number of players. Comparative statics with respect to changes in players' values are also derived. Section 4 analyzes the efficiency properties of equilibrium, and Section 5 concludes.

\section{The subscription game with threshold uncertainty}

We consider the problem of $n$ players who simultaneously contribute any positive amount to the funding of a binary public good. Player $i$ 's value for the good is $v_{i}, i=1, \ldots, n$. Players' values $v_{i}$ are independently distributed random variables with cumulative distribution functions (cdf) $F_{i}$, which have supports $\left[\underline{v}_{i}, \bar{v}_{i}\right]$, where $0 \leq \underline{v}_{i}<\bar{v}_{i}<\infty, i=1, \ldots, n$. A player's realized value is known only to that player. We suppose $F_{i}$ is absolutely continuous, with density function $f_{i}$. The cost of the public good is $c$, distributed with absolutely

\footnotetext{
${ }^{2}$ McBride assumes players' contribution choice is binary - individuals contribute either $c$ or nothing.
} 
continuous cdf $H$ having density $h$ on $[\underline{c}, \bar{c}]$, where $0 \leq \underline{c}<\sum \bar{v}_{i} \leq \bar{c}$ (without index, the summation $\sum$ is over the entire player set $i=1, \ldots, n)$.

In the terminology of Admati and Perry (1991), we consider the subscription game: players' contributions are refunded if they are insufficient to cover $c$. If the good is provided, then the payoff to player $i$ is $v_{i}$ - (player $i$ 's contribution). If the good is not provided, then the payoff to player $i$ is 0 . Therefore, the expected utility of agent $i$ with value $v_{i}$ contributing $x$ is

$$
U_{i}\left(x \mid v_{i}\right) \equiv\left(v_{i}-x\right) \operatorname{Pr}\left(x+\sum_{j \neq i} s_{j}\left(v_{j}\right) \geq c\right)
$$

where $s_{j}$ denotes agent $j$ 's strategy, for $j=1, \ldots, n$. For any vector $v=\left(v_{1}, \ldots, v_{n}\right)$ we denote by $v_{-i}$ the vector $\left(v_{1}, \ldots, v_{i-1}, v_{i+1}, \ldots, v_{n}\right)$ and denote its joint distribution by $F_{-i}$. We similarly define $\underline{v}_{-i}$ and $\bar{v}_{-i}$. Now $U_{i}$ in (1) can be expressed as

$$
U_{i}\left(x \mid v_{i}\right)=\int_{\underline{v}_{-i}}^{\bar{v}_{-i}}\left(v_{i}-x\right) H\left(x+\sum_{j \neq i} s_{j}\left(v_{j}\right)\right) d F_{-i}\left(v_{-i}\right) .
$$

Corollary 2.1 in Athey (2001) yields the following existence result.

Proposition 1 (Existence of a pure-strategy equilibrium in increasing strategies). Suppose the densities $f_{i}$, $i=1, \ldots, n$, are bounded. Then for any continuous distribution of the cost $H$, there exists a pure-strategy equilibrium in increasing strategies.

The proof of Proposition 1, a verification of the conditions of Athey's corollary, is given in the Appendix.

We next provide conditions under which the equilibrium in the subscription game is unique. To do this we assume $\underline{c}=0$. This assumption implies that any contribution larger than $v_{i}$ is strictly dominated by a contribution of $v_{i}$, so without loss of generality we restrict attention to contributions in $\left[0, \bar{v}_{i}\right]$ for any player $i$. Now, if the threshold distribution $H$ is concave, then $U_{i}$ in (2) is strictly quasi-concave in $x$, because of the assumption $\bar{c} \geq \sum \bar{v}_{i}$ (see below). Standard results then imply uniqueness of the optimal contribution for each type, given any profile of contributions for the other players. In particular, the derivative of $U_{i}$ with respect to $x$ is

$$
\begin{aligned}
\frac{\partial U_{i}\left(x \mid v_{i}\right)}{\partial x} & =\left(v_{i}-x\right) \int_{\underline{v}_{-i}}^{\bar{v}_{-i}} h\left(x+\sum_{j \neq i} s_{j}\left(v_{j}\right)\right) d F_{-i}\left(v_{-i}\right)-\int_{\underline{v}_{-i}}^{\bar{v}_{-i}} H\left(x+\sum_{j \neq i} s_{j}\left(v_{j}\right)\right) d F_{-i}\left(v_{-i}\right) \\
& =\left[\int_{\underline{v}_{-i}}^{\bar{v}_{-i}} h\left(x+\sum_{j \neq i} s_{j}\left(v_{j}\right)\right) d F_{-i}\left(v_{-i}\right)\right] \times\left[v_{i}-x-r_{i}\left(x, \sum_{j \neq i} s_{j}\right)\right],
\end{aligned}
$$


where $r_{i}$ is defined as the ratio

$$
r_{i}\left(x, \sum_{j \neq i} s_{j}\right) \equiv \frac{\int_{\underline{v}_{-i}}^{\bar{v}_{-i}} H\left(x+\sum_{j \neq i} s_{j}\left(v_{j}\right)\right) d F_{-i}\left(v_{-i}\right)}{\int_{\underline{v}_{-i}}^{\bar{v}_{-i}} h\left(x+\sum_{j \neq i} s_{j}\left(v_{j}\right)\right) d F_{-i}\left(v_{-i}\right)} .
$$

Note that $r_{i}$ is well-defined because the strict dominance argument above implies the denominator is nonzero. Moreover, when $H$ is concave, $r_{i}$ is strictly increasing in each of its arguments, so by (3) $U_{i}$ is indeed quasiconcave in $x$. The first-order conditions imply that if $x^{*}$ is the best response of agent $i$ with type $v_{i}$, then $x^{*}$ must solve the following equation in $x$,

$$
x=\max \left\{v_{i}-r_{i}\left(x, \sum_{j \neq i} s_{j}\right), 0\right\} .
$$

The determination of the unique optimal (positive) contribution level, using equation (5), is depicted in Figure 1.

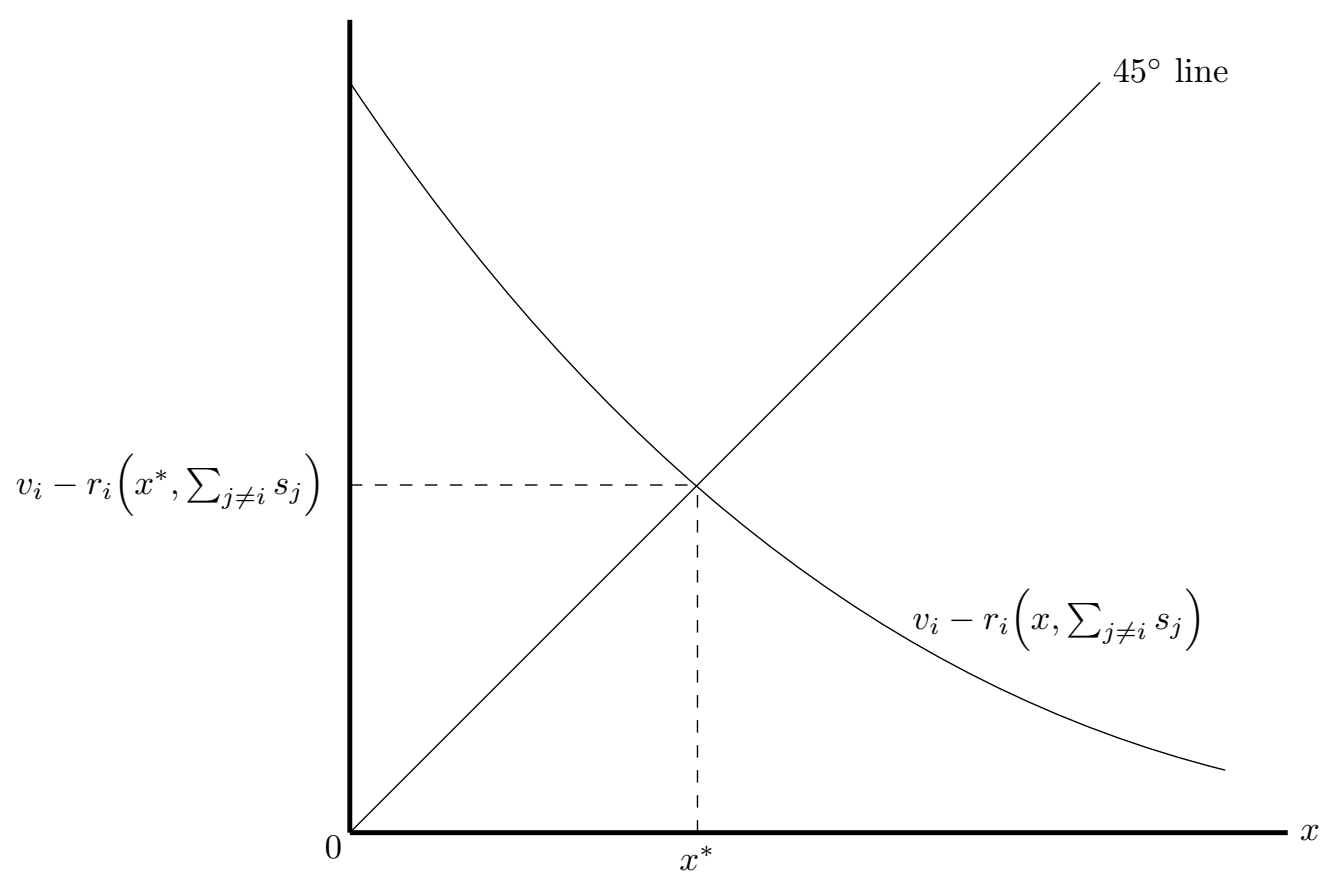

Figure 1: Determining the best response of player $i$ with value $v_{i}$

Beyond providing a graphical proof of the uniqueness of each type's best-response contribution level, Figure 1 also shows why once a player's contribution is positive, it is strictly increasing for all larger values: because increases in value simply shift upward the $v_{i}-r_{i}$ curve, the intersection with the $45^{\circ}$ line occurs at a larger contribution level. 
Before proceeding, we note that equilibrium strategies are almost everywhere differentiable, and it is convenient to establish the following fact. For any $v_{i}$ at which the equilibrium contribution function $s_{i}$ is strictly positive and differentiable, the derivative does not exceed $\frac{1}{2}$. To see this last point, note that $s_{i}\left(v_{i}\right)>0$ implies $s_{i}\left(v_{i}^{\prime}\right)>s_{i}\left(v_{i}\right)>0$ for any $v_{i}^{\prime}>v_{i}$, so, denoting $s_{i}\left(v_{i}^{\prime}\right)-s_{i}\left(v_{i}\right)$ with $\Delta_{s}$, we have

$$
\begin{array}{rlr}
\Delta_{s} & =\left(v_{i}^{\prime}-v_{i}\right)-\left[r_{i}\left(s_{i}\left(v_{i}^{\prime}\right), \sum_{j \neq i} s_{j}\right)-r_{i}\left(s_{i}\left(v_{i}\right), \sum_{j \neq i} s_{j}\right)\right] & \text { (by (5)) } \\
& \leq\left(v_{i}^{\prime}-v_{i}\right)-\frac{\int_{\underline{v}_{-i}}^{\bar{v}_{-i}}\left[H\left(s_{i}\left(v_{i}^{\prime}\right)+\sum_{j \neq i} s_{j}\left(v_{j}\right)\right)-H\left(s_{i}\left(v_{i}\right)+\sum_{j \neq i} s_{j}\left(v_{j}\right)\right)\right] d F_{-i}\left(v_{-i}\right)}{\int_{\underline{v}_{-i}}^{\bar{v}_{-i}} h\left(s_{i}\left(v_{i}^{\prime}\right)+\sum_{j \neq i} s_{j}\left(v_{j}\right)\right) d F_{-i}\left(v_{-i}\right)} & \text { (by } h \text { decreasing) } \\
& \leq\left(v_{i}^{\prime}-v_{i}\right)-\Delta_{s}, & \text { (by concavity of } H \text { ) }
\end{array}
$$

from which the desired result follows immediately. Therefore, the equilibrium contribution $s_{i}\left(v_{i}\right)$ of type $v_{i}$ cannot exceed $\frac{1}{2}\left(\underline{v}_{i}+v_{i}\right)$.

We next establish that, when there are two players, equilibrium in the subscription game is unique. This uniqueness stands in contrast to the subscription game with fixed threshold, where a continuum of equilibria is the norm.

Proposition 2 (Uniqueness of equilibrium). Suppose $H$ is a concave distribution with support $[0, \bar{c}]$. Furthermore, assume $\bar{c} \geq \bar{v}_{1}+\bar{v}_{2}+\max \left\{\underline{v}_{1}, \underline{v}_{2}\right\}$. Then the two-player subscription game with threshold uncertainty has a unique equilibrium.

Proof. With $H$ concave, condition (5) must hold, so any equilibrium $\left(s_{1}, s_{2}\right)$ must satisfy

$$
s_{1}\left(v_{1}\right)=\max \left\{v_{1}-r_{1}\left(s_{1}\left(v_{1}\right), s_{2}\right), 0\right\}
$$

and

$$
s_{2}\left(v_{2}\right)=\max \left\{v_{2}-r_{2}\left(s_{2}\left(v_{2}\right), s_{1}\right), 0\right\} .
$$

Equation (6) implicitly defines the operator $T_{1}\left(s_{2}\right)$, where the value of $T_{1}\left(s_{2}\right)\left(v_{1}\right)$ is given by the solution for $x^{*}$ of (5). Similarly, (7) defines an operator $T_{2}\left(s_{1}\right)$, with $T_{2}\left(s_{1}\right)\left(v_{2}\right)$ also determined via (5). Therefore, defining the operators $O_{1}\left(s_{1}\right) \equiv T_{1}\left(T_{2}\left(s_{1}\right)\right)$ and $O_{2}\left(s_{2}\right) \equiv T_{2}\left(T_{1}\left(s_{2}\right)\right)$, any equilibrium $\left(s_{1}, s_{2}\right)$ must be such that $s_{1}$ is a fixed point of $O_{1}$ and $s_{2}$ is a fixed point of $O_{2}$. By way of contradiction, suppose two equilibria exist: $\left(s_{1}, s_{2}\right)$ and $\left(\tilde{s}_{1}, \tilde{s}_{2}\right)$. To show uniqueness of equilibrium, we proceed to show $O_{i}$ satisfies

$$
d\left(O_{i}\left(s_{i}\right), O_{i}\left(\tilde{s}_{i}\right)\right)<d\left(s_{i}, \tilde{s}_{i}\right) \text { if } d\left(s_{i}, \tilde{s}_{i}\right)>0
$$


where $d\left(s_{i}, \tilde{s}_{i}\right) \equiv \sup _{v_{i}}\left|s_{i}\left(v_{i}\right)-\tilde{s}_{i}\left(v_{i}\right)\right|$. We prove (8) for $O_{1}$ only, because the proof for $O_{2}$ is identical, and for brevity, we indicate $d\left(s_{1}, \tilde{s}_{1}\right)$ with $d$, which we assume strictly positive. The steps of our proof mirror Blackwell's (1965) sufficient conditions for a function to be a contraction mapping. ${ }^{3}$ We first prove that $O_{1}$ satisfies the following limited form of "monotonicity":

$$
O_{1}\left(\tilde{s}_{1}+d\right)\left(v_{1}\right) \geq O_{1}\left(s_{1}\right)\left(v_{1}\right), \forall v_{1}
$$

we then show the following version of "discounting":

$$
O_{1}\left(\tilde{s}_{1}+d\right)\left(v_{1}\right)<O_{1}\left(\tilde{s}_{1}\right)\left(v_{1}\right)+d, \forall v_{1}
$$

Condition (9) follows because the ratios $r_{i}$, defined in (4) and used in $O_{1}$, are strictly increasing in each argument, due to the concavity of $H$. Therefore, each operator $T_{i}$ is decreasing in its function argument, so that $O_{1}$, the application of $T_{1}$ to $T_{2}\left(s_{1}\right)$, is decreasing in $T_{2}\left(s_{1}\right)$; and ultimately $O_{1}$ is increasing in its function argument $s_{1}$. The formal verification of this argument appears in the following lemma, with proof in the Appendix.

Lemma 1 (Monotonicity). $O_{1}\left(\tilde{s}_{1}+d\right)\left(v_{1}\right) \geq O_{1}\left(s_{1}\right)\left(v_{1}\right), \forall v_{1}$.

We establish (10) using the following relationships: first

$$
T_{2}\left(\tilde{s}_{1}+d\right)\left(v_{2}\right)>T_{2}\left(\tilde{s}_{1}\right)\left(v_{2}\right)-d
$$

and then

$$
T_{1}\left(T_{2}\left(\tilde{s}_{1}+d\right)\right)\left(v_{1}\right)<T_{1}\left(T_{2}\left(\tilde{s}_{1}\right)\right)\left(v_{1}\right)+d .
$$

Condition (11) says that if for all values player 1 increases his contribution by $d$, then player 2's best response is to reduce his contributions by less than $d$; and (12) says that a further iteration of the best-response operator results in player 1 increasing his contributions only by less than $d$. A relationship analogous to (11) also holds for $T_{1}$, from which (12) then follows straightforwardly. Therefore, the key intermediate step in establishing monotonicity of $O_{1}$ is to establish (11). Clearly (11) is satisfied if $T_{2}\left(\tilde{s}_{1}\right)\left(v_{2}\right)=0$, so it only remains to consider the case of $T_{2}\left(\tilde{s}_{1}\right)\left(v_{2}\right)>0$, as depicted in Figure 2 .

\footnotetext{
${ }^{3}$ The inequality in (8) requires that, when applied to two prospective equilibrium strategies, $O_{i}$ is a contraction. The usual contraction mapping condition requires that, for some $\beta<1$, it is the case that $d\left(O_{i}(s), O_{i}(\tilde{s})\right)<\beta d(s, \tilde{s})$ for any pair of distinct functions $s, \tilde{s}$. This stricter bound is generally used to establish existence of a fixed point as well as uniqueness. We do not need to establish existence of an equilibrium - at least one exists by Proposition 1.
} 


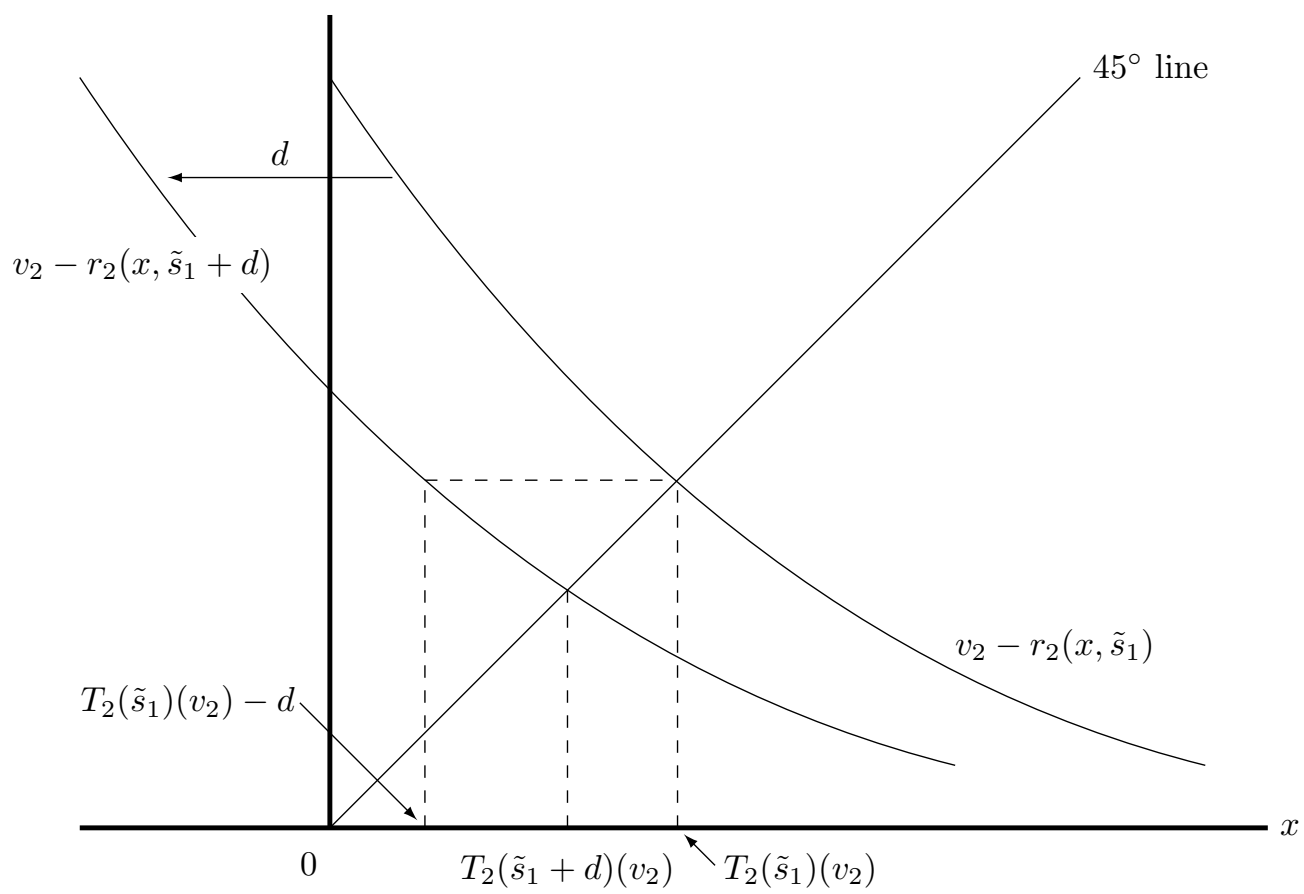

Figure 2: Intermediate step in establishing that the operator $O_{i}$ exhibits "discounting"

Observe from (4) that $r_{2}\left(x, \tilde{s}_{1}+d\right)=r_{2}\left(x+d, \tilde{s}_{1}\right)$, so that the curve labelled $v_{2}-r_{2}\left(x, \tilde{s}_{1}+d\right)$ is simply a leftward translation of the original curve, labelled $v_{2}-r_{2}\left(x, \tilde{s}_{1}\right)$, by the amount $d$. Because these curves are downward sloping, it follows, as shown in Figure 2, that $T_{2}\left(\tilde{s}_{1}\right)\left(v_{2}\right)-d<T_{2}\left(\tilde{s}_{1}+d\right)\left(v_{2}\right)$, which is (11). Equation (12) is demonstrated along similar lines. The proof of the following lemma establishes (11) and (12), and thereby (10), in the Appendix.

Lemma 2 (Discounting). $O_{1}\left(\tilde{s}_{1}+d\right)\left(v_{1}\right)<O_{1}\left(\tilde{s}_{1}\right)\left(v_{1}\right)+d, \forall v_{1}$.

Following Blackwell (1965) we have, for the two equilibrium strategies $s_{1}$ and $\tilde{s}_{1}$,

$$
O_{1}\left(s_{1}\right) \leq O_{1}\left(\tilde{s}_{1}+d\left(s_{1}, \tilde{s}_{1}\right)\right)<O_{1}\left(\tilde{s}_{1}\right)+d\left(s_{1}, \tilde{s}_{1}\right)
$$

where the first inequality follows from monotonicity and the second from discounting; similarly,

$$
O_{1}\left(\tilde{s}_{1}\right) \leq O_{1}\left(s_{1}+d\left(s_{1}, \tilde{s}_{1}\right)\right)<O_{1}\left(s_{1}\right)+d\left(s_{1}, \tilde{s}_{1}\right) .
$$

Combining these results yields (8). Because there are two distinct equilibria, $\left(s_{1}, s_{2}\right)$ and $\left(\tilde{s}_{1}, \tilde{s}_{2}\right)$, either $s_{1} \neq \tilde{s}_{1}$ or $s_{2} \neq \tilde{s}_{2}$. If $s_{1} \neq \tilde{s}_{1}$, then

$$
0<d\left(s_{1}, \tilde{s}_{1}\right)=d\left(O_{1}\left(s_{1}\right), O_{1}\left(\tilde{s}_{1}\right)\right)<d\left(s_{1}, \tilde{s}_{1}\right)
$$


this contradiction shows it must be that $s_{1}=\tilde{s}_{1}$. Similarly, it cannot be that $s_{2} \neq \tilde{s}_{2}$. Therefore, there cannot exist two distinct equilibria. Hence, the equilibrium, whose existence is assured by Proposition 1, must be unique.

As a technical remark, it is worth noting that the stronger assumption $\bar{c} \geq \bar{v}_{1}+\bar{v}_{2}+\max \left\{\underline{v}_{1}, \underline{v}_{2}\right\}$ in Proposition 2, rather than $\bar{c} \geq \bar{v}_{1}+\bar{v}_{2}$ as in the earlier part of this section, is only for ease of exposition. It ensures that the denominator of $r_{i}$ in (4) remains non-zero even when, in the course of the proof, the distance $d$ is added to an equilibrium strategy. Proposition 2 can be proved under the weaker assumption that $\bar{c} \geq \bar{v}_{1}+\bar{v}_{2}$ by expanding, as follows, the definition of the ratio $r_{i}$ in (4) when the denominator is zero: set $r_{i}$ to any value larger than $\bar{v}_{i}$. At the cost of more cumbersome notation, all steps in the proof go through essentially unchanged.

\section{A uniformly distributed threshold}

In this section we assume the threshold cost is uniformly distributed on $[0, \bar{c}]$. With this assumption, the subscription game with an uncertain threshold becomes simpler to analyze, and several interesting results follow. Given $H(c)=c / \bar{c}$, in (2) the probability that the good is provided is $\mathrm{E}\left[H\left(x+\sum_{j \neq i} s_{j}\left(v_{j}\right)\right) \mid x, v_{i}\right]=$ $\frac{1}{\bar{c}} \mathrm{E}\left[x+\sum_{j \neq i} s_{j}\left(v_{j}\right) \mid x, v_{i}\right]$, so the utility of agent $i,(2)$, becomes

$$
U_{i}\left(x \mid v_{i}\right)=\frac{1}{\bar{c}}\left(v_{i}-x\right)\left(x+\sum_{j \neq i} K_{j}\right)
$$

where $K_{j} \equiv \mathrm{E}\left[s_{j}\left(v_{j}\right)\right]$, for $j=1, \ldots, n$; that is, $K_{j}$ is the expected contribution of agent $j$. The comparison of equations (2) and (13) immediately reveals the simplification that the uniform distribution assumption yields. Without it, in general, an agent needs to forecast the whole distribution of all of his rivals' strategies, see (2). With a uniformly distributed threshold, an agent needs to forecast only the expected value of his rivals' strategies, see (13).

We now proceed to the characterization of equilibria. Since $U_{i}$ in (13) is strictly concave in $x$, the firstderivative $\frac{\partial}{\partial x} U_{i}\left(x \mid v_{i}\right)=\frac{1}{\bar{c}}\left(v_{i}-\sum_{j \neq i} K_{j}-2 x\right)$, along with the non-negativity constraint on $x$, yields the following "best-response" function for player $i$,

$$
s_{i}\left(v_{i} \mid \sum_{j \neq i} K_{j}\right)=\max \left\{0, \frac{1}{2}\left(v_{i}-\sum_{j \neq i} K_{j}\right)\right\} .
$$

Therefore, using the definition of $K_{i}$ above and (14), in equilibrium the following system of equations must be satisfied by $\left(K_{1}, \ldots, K_{n}\right)$ : 


$$
K_{i}=\mathrm{E}\left[s_{i}\left(v_{i} \mid \sum_{j \neq i} K_{j}\right)\right], \quad \forall i
$$

For general concave threshold distributions with support $[0, \bar{c}]$, Proposition 2 established uniqueness of equilibrium only for the case of two contributors. Additionally requiring this distribution to be uniform, we next establish uniqueness of equilibrium for any number of players. The following lemma, whose proof is in the Appendix, demonstrates that there exists at most one solution to the system of equations in (15).

Lemma 3 (Uniqueness). There exists at most one solution $\left(K_{1}^{*}, \ldots, K_{n}^{*}\right)$ to the system of equations (15).

Collecting the results of our previous analysis, we obtain the following.

Proposition 3 (Uniqueness and Characterization of Equilibrium). In the subscription game with threshold uncertainty, there exists a unique equilibrium; the equilibrium strategy, $s_{i}^{*}$, for agent $i$ is $s_{i}\left(v_{i} \mid \sum_{j \neq i} K_{j}^{*}\right)$ as given in (14) and $\left(K_{1}^{*}, \ldots, K_{n}^{*}\right)$ denotes the unique solution of (15). The ex ante equilibrium probability that the good is provided equals $\frac{1}{\bar{c}} \sum K_{i}^{*}$.

Proof. Proposition 1 guarantees at least one equilibrium exists. The analysis above shows any equilibrium must have strategies given by (14), for some set of numbers $K_{1}, \ldots, K_{n}$. The system (14) implies (15), and Lemma 3 implies that there is a unique solution to (15). Therefore, there is a unique profile of equilibrium strategies.

Because $c$ is uniformly distributed on $[0, \bar{c}]$, for any given vector of values $v=\left(v_{1}, \ldots, v_{n}\right)$, the probability of provision is $H\left(\sum s_{i}^{*}\left(v_{i}\right)\right)=\frac{1}{\bar{c}} \sum s_{i}^{*}\left(v_{i}\right)$. Therefore, the ex ante probability of provision is $\mathrm{E}\left[H\left(\sum s_{i}^{*}\left(v_{i}\right)\right)\right]=$ $\frac{1}{\bar{c}} \sum \mathrm{E}\left[s_{i}^{*}\left(v_{i}\right)\right]=\frac{1}{\bar{c}} \sum K_{i}^{*}$.

The following examples illustrate equilibrium in the subscription game with threshold uncertainty.

Example 1 (Values are uniformly distributed between 0 and 1).

Consider two players and suppose values are independently and uniformly distributed on $[0,1]$. Then both players use the same strategy and (15) reduces to

$$
K=\int_{K}^{1} \frac{1}{2}(v-K) d v=\frac{1}{4}-\frac{K}{2}+\frac{K^{2}}{4}
$$

the solution to which is $K^{*}=3-2 \sqrt{2} \approx 0.17157$. Therefore, the corresponding symmetric equilibrium strategy is

$$
s_{i}^{*}(v)= \begin{cases}\frac{1}{2}\left(v_{i}-3+2 \sqrt{2}\right) & \text { if } v_{i} \geq 3-2 \sqrt{2} \\ 0 & \text { if } v_{i}<3-2 \sqrt{2} .\end{cases}
$$


Ex post efficiency would require that the public good be provided if and only if $v_{1}+v_{2} \geq c$. If, further, $c$ is uniformly distributed over $[0,2]$ and all random variables are independent, then the good would efficiently be provided with probability 0.5 . However, by Proposition 3 the subscription-game equilibrium provides the good only with probability $2 K^{*} / \bar{c}=3-2 \sqrt{2} \approx 0.17157$, or in about $34 \%$ of the cases where it should be provided.

As Example 1 clearly illustrates, and in sharp contrast with the known-threshold case, ${ }^{4}$ equilibrium is unique and tractable, thus making our environment useful for understanding design features of the subscription game. For instance, it has sometimes been suggested that allowing players to make contributions of any amount, rather than simply one of a preselected set, can increase overall contributions and thereby the likelihood of provision. Cadsby and Maynes (1999) provide an experiment testing allowing continuous or "all-or-nothing" contribution possibilities and find that the continuous case yielded greater contributions. Their finding that one particular specification of discrete contribution levels yields less than the continuous possibilities case is interesting but hardly conclusive - perhaps other specifications of the binary possibilities could do better. Our framework is ideal for formulating this comparison. We do this next.

Example 2 (The probability of provision: binary or continuous contribution possibilities).

We again suppose there are two players, values are independently and uniformly distributed on $[0,1]$, and that cost is uniformly distributed over $[0, \bar{c}]$, where $\bar{c} \geq 2$. From Proposition 3 and Example 1 we know that each player's expected contribution in the continuous-contribution case is $K^{*}=3-2 \sqrt{2}$.

Next suppose that players' contributions are restricted to be either 0 or $b$, (we may assume $b \leq 1$ ). Equilibrium has players use a strategy given by

$$
s(v)= \begin{cases}b & \text { if } v \geq v^{*} \\ 0 & \text { if } v<v^{*}\end{cases}
$$

for some critical value $v^{*}$ that depends on $b$. Player 1 with value $v$ contributing 0 earns payoff

$$
U\left(0 \mid v_{1}\right)=\operatorname{Pr}\left(v_{2} \geq v^{*}\right) \operatorname{Pr}(c \leq b) v_{1}=\left(1-v^{*}\right) \frac{b}{\bar{c}} v_{1}
$$

if instead player 1 contributes $b$, his payoff is

$$
\begin{aligned}
U\left(b \mid v_{1}\right) & =\operatorname{Pr}\left(v_{2}<v^{*}\right) \operatorname{Pr}(c \leq b)\left(v_{1}-b\right)+\operatorname{Pr}\left(v_{2} \geq v^{*}\right) \operatorname{Pr}(c \leq 2 b)\left(v_{1}-b\right) \\
& =\left(v^{*} \frac{b}{\bar{c}}+\left(1-v^{*}\right) \frac{2 b}{\bar{c}}\right)\left(v_{1}-b\right) .
\end{aligned}
$$

\footnotetext{
${ }^{4}$ See, for example, Alboth et al. (2001) for a description of the case where agents' values are uniformly distributed.
} 
The threshold value $v^{*}$ denotes the type indifferent between contributing 0 and $b$; solving $U(0 \mid v)=U(b \mid v)$ for $v$ we obtain

$$
v^{*}(b)=\frac{2 b}{1+b} .
$$

Each player's expected contribution is $K(b) \equiv b \operatorname{Pr}\left(v \geq v^{*}(b)\right)=\left(\frac{1-b}{1+b}\right) b$. This expected contribution is strictly quasiconcave in $b$, reaching its maximum at $b^{*}=\sqrt{2}-1$; the resulting expected contribution of each player is

$$
K\left(b^{*}\right)=\frac{(2-\sqrt{2})(\sqrt{2}-1)}{\sqrt{2}}=3-2 \sqrt{2},
$$

which is precisely the same expected contribution as in the continuous-case model. Thus, the probability of provision in the two settings is also identical. Note, though, for any choice of $b$ other than $b^{*}$, the binary-contribution model yields strictly lower contributions.

The next example shows our framework may be used to study how the equilibrium of the subscription game varies as the distributions of players' values change.

Example 3 (Asymmetric players with values related by stochastic dominance).

In this example players 1 and 2 are not symmetric. As in Example 1, we continue to assume that the distribution of player 2's value, $F_{2}$, is uniform over [0,1], but for player 1 we deviate from Example 1 in two natural ways: we consider distributions that are related to the uniform distribution by either first-order or second-order stochastic dominance. We continue to assume the support of player 1 's value is $[0,1]$, but in the first instance it has $\operatorname{cdf} \hat{F}_{1}(v)=1-(1-v)^{2}$ and in the second it has cdf $\tilde{F}_{1}(v)=3 v^{2}-2 v^{3}$. Letting $F_{1}$ denote the uniform distribution over $[0,1]$ in Example 1, it is readily verified that $F_{1}$ first-order stochastically dominates $\hat{F}_{1}$ and $\tilde{F}_{1}$ second-order stochastically dominates $F_{1}$. Figure 3 depicts these cdfs.

Table 1: Expected equilibrium contributions when players' values are distributed on $[0,1]$ with $v_{2}$ being uniformly distributed and $v_{1}$ having the alternative distributions shown.

\begin{tabular}{l|c|c|c|} 
& $K_{1}^{*}$ & $K_{2}^{*}$ & $K_{1}^{*}+K_{2}^{*}$ \\
\hline \hline$F_{1}(v)=v$ & 0.17157 & 0.17157 & 0.34314 \\
\hline$\hat{F}_{1}(v)=1-(1-v)^{2}$ & 0.08195 & 0.21070 & 0.29265 \\
\hline$\tilde{F}_{1}(v)=3 v^{2}-2 v^{3}$ & 0.16533 & 0.17417 & 0.33950 \\
\hline
\end{tabular}

Table 1 shows the resulting expected efforts of the two players for the respective pairs of distributions. ${ }^{5}$ Consider first the effect of moving from the pair $\left(F_{1}, F_{2}\right)$ to $\left(\hat{F}_{1}, F_{2}\right)$, according to which player 1 's values

\footnotetext{
${ }^{5}$ Derivations of equilibrium calculations are available from the authors.
} 
are reduced in the sense of first-order stochastic dominance. We see that player 1's expected contribution falls and player 2's increases. Indeed, the change from $F_{1}$ to $\hat{F}_{1}$ results in a weak reduction in player 1's contribution strategy and a weak increase in player 2's. ${ }^{6}$ This effect is very intuitive. When player 1's distribution of values is shifted leftward, player 2 recognizes that, even if player 1 were not to change his contribution strategy, player 1's expected contribution would fall, and this induces player 2 to increase his contributions. This induced increase in player 2's contributions then actually induces player 1 to reduce his contribution strategy, reinforcing player 2's need to increase his own contribution. In the new equilibrium, player 1's contribution strategy has decreased and player 2's has increased.

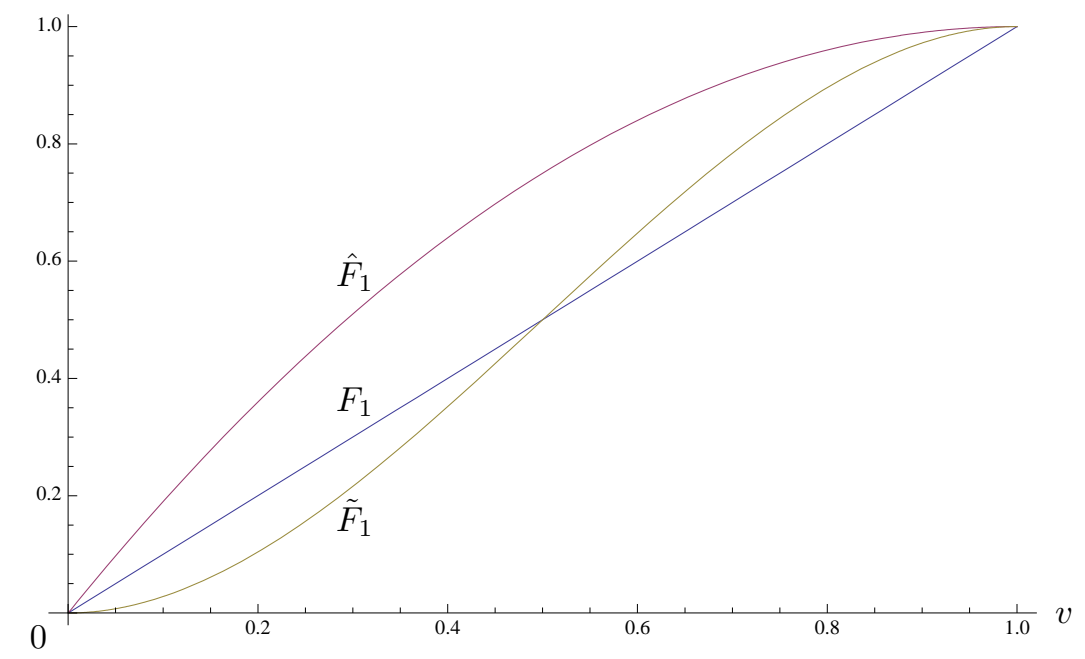

Figure 3: Value cdfs in Example 3

Next consider the effect of moving from the pair $\left(F_{1}, F_{2}\right)$ to $\left(\tilde{F}_{1}, F_{2}\right)$, according to which player 1's values become less dispersed around the mean $\left(F_{1}\right.$ is a mean-preserving spread of $\left.\tilde{F}_{1}\right)$. Again we see the change from $F_{1}$ to $\tilde{F}_{1}$ results in a weak reduction in player 1's contribution strategy and a weak increase in player 2's. Here, too, even if player 1 were not to change his equilibrium contribution strategy, his average contribution would fall because his strategy is a convex function of his value and $\tilde{F}_{1}$ second-order stochastically dominates $F_{1}$. Given this reduction in player 1's expected contribution, player 2 is induced to increase his contributions, and this in turn induces player 1 to reduce his own contribution strategy. The net result is that player 1's strategy is weakly reduced and player 2's is weakly increased.

The relationships among contribution strategies in Example 3 hold in general, as we will show. Before doing that, it is convenient to establish a general result on the crowding-out by exogenous contributions to the public good.

\footnotetext{
${ }^{6}$ Player 1's reduction is strict over the range of values where his contribution is strictly positive under $F_{1}$ and player 2 's increase is strict over the range where his contribution is strictly positive when player 1 has cdf $\hat{F}_{1}$.
} 


\subsection{Crowding-out}

We denote with $K^{E}$ the level of contributions that are exogenously provided by an external authority; and we consider how the players' contributions change as $K^{E}$ increases. It is immediate to replicate the steps leading to Proposition 3 and obtain that in equilibrium, the system of equations (15) takes the form

$$
\forall i, 0=K_{i}-\frac{1}{2} \int_{\sum_{j \neq i} K_{j}+K^{E}}^{\bar{v}_{i}}\left(v_{i}-\sum_{j \neq i} K_{j}-K^{E}\right) f_{i}\left(v_{i}\right) d v_{i} .
$$

The application of the implicit function theorem to (16) leads to the following proposition (the proof is in the Appendix).

Proposition 4 (Crowding out). Define $K^{N} \equiv \sum K_{j}$ and suppose $K^{E}+\sum \bar{v}_{i}<\bar{c}$. Then

a. $d K_{i} / d K^{E}<0$ for all $i$ such that $K_{i}>0$;

b. $-\frac{n}{n+1}<\frac{d K^{N}}{d K^{E}}<0$.

Beyond establishing, in part b, that crowding-out is only partial in our model, Proposition 4 allows an interesting graphical analysis of the equilibrium system of equations (15), even when the number of players is larger than 2. It is indeed possible to separate out player 1 from the aggregate of all other players, and represent expected contributions on a Cartesian plane. Letting $K_{-1} \equiv \sum_{j \neq 1} K_{j}$, from (14) and (15), we define the following "best-response" function for player 1 ,

$$
K_{1}=R_{1}\left(K_{-1}\right) \equiv \frac{1}{2} \int_{K_{-1}}^{\bar{v}_{1}}\left(v-K_{-1}\right) f_{1}(v) d v
$$

for given $K_{-1}$. Observe that the function $R_{1}$ is continuous, convex, and strictly decreasing, with slope less than $1 / 2$ in absolute value. Similarly, we can consider all equations in (14) and (15), except the one for player 1, as defining a "best-response" function for all other players, given some exogenously fixed level for $K_{1}$. The sum of all solutions $K_{2}, \ldots, K_{n}$ then can be written as $R_{-1}\left(K_{1}\right)$ and, in equilibrium $K_{-1}=R_{-1}\left(K_{1}\right)$. By Proposition 4, with $K_{1}$ assuming the role of $K^{E}$, we also see $R_{-1}$ is strictly decreasing, with slope less than $(n-1) / n<1$ in absolute value. The unique equilibrium occurs at the intersection of $R_{1}$ and $R_{-1}$, as Figure 4 illustrates. This figure is useful in the analysis of perturbations of the distribution of values, which we undertake next.

\subsection{Stochastic dominance}

We first consider distributions $F_{1}, F_{2}, \ldots, F_{n}$ for players' values, and denote the unique expected equilibrium contributions from $(15)$ as $K_{1}^{*}, K_{2}^{*}, \ldots, K_{n}^{*}$. We then consider the distributions $\hat{F}_{1}, F_{2}, \ldots, F_{n}$, where $F_{2}, \ldots, F_{n}$ 


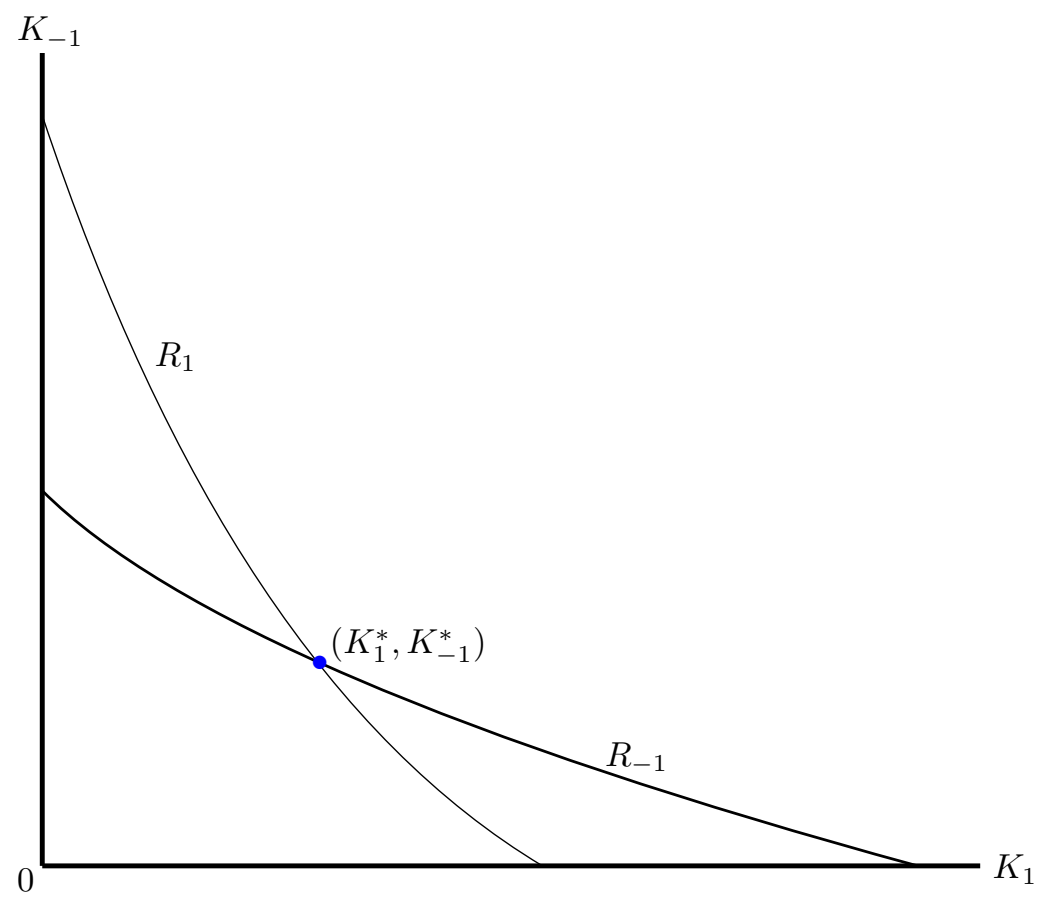

Figure 4: Illustration of equilibrium

remain the same, but either $F_{1}$ first-order stochastically dominates (FOSD) $\hat{F}_{1}$ or $\hat{F}_{1}$ second-order stochastically dominates $(\mathrm{SOSD}) F_{1}$. We denote the expected equilibrium contributions in this case as $\hat{K}_{1}^{*}, \hat{K}_{2}^{*}, \ldots, \hat{K}_{n}^{*}$. We have the following results.

Proposition 5 (Stochastic dominance). Fix the distributions $F_{2}, \ldots, F_{n}$. Consider two distributions for agent 1 's values, $F_{1}$ and $\hat{F}_{1}$. If either $F_{1}$ first-order stochastically dominates $\hat{F}_{1}$ or $\hat{F}_{1}$ second-order stochastically dominates $F_{1}$, then

a. $\hat{K}_{1}^{*} \leq K_{1}^{*}$ and $\hat{K}_{j}^{*} \geq K_{j}^{*}, \forall j \geq 2$ : Agent 1 contributes less on average and every other agent contributes more on average when the distributions of values are $\left(\hat{F}_{1}, F_{2}, \ldots, F_{n}\right)$ than when they are $\left(F_{1}, F_{2}, \ldots, F_{n}\right)$;

b. $\sum \hat{K}_{i}^{*} \leq \sum K_{i}^{*}$ : Total expected contributions are smaller when the distributions of values are $\left(\hat{F}_{1}, F_{2}, \ldots, F_{n}\right)$ than when they are $\left(F_{1}, F_{2}, \ldots, F_{n}\right)$;

c. The equilibrium payoff of each type of agent 1 is at least as great when the distributions of values are $\left(\hat{F}_{1}, F_{2}, \ldots, F_{n}\right)$ as when they are $\left(F_{1}, F_{2}, \ldots, F_{n}\right)$;

d. The equilibrium payoff of each type of agent $j$, with $j \geq 2$, is no greater when the distributions of values are $\left(\hat{F}_{1}, F_{2}, \ldots, F_{n}\right)$ than when they are $\left(F_{1}, F_{2}, \ldots, F_{n}\right)$.

Proof. To establish part a, denote by $R_{1}\left(K_{-1} \mid F_{1}\right)$ the function in (17) when player 1's distribution of values is $F_{1}$ and denote by $R_{1}\left(K_{-1} \mid \hat{F}_{1}\right)$ the function in (17) when player 1 's distribution of values is $\hat{F}_{1}$. Recall 
that if $F_{1}$ first-order stochastically dominates $\hat{F}_{1}$, then the expected value of any increasing function of $v_{1}$ is at least as large under $F_{1}$ as under $\hat{F}_{1}$; recall too that if $\hat{F}_{1}$ second-order stochastically dominates $F_{1}$, then the expected value of any convex function of $v_{1}$ is at least as large under $F_{1}$ as under $\hat{F}_{1}$. Therefore,

$$
\begin{aligned}
R_{1}\left(K_{-1} \mid \hat{F}_{1}\right) & =\frac{1}{2} \int_{\underline{v}_{1}}^{\bar{v}_{1}} \max \left\{0, v_{1}-K_{-1}\right\} d \hat{F}_{1}\left(v_{1}\right) \\
& \leq \frac{1}{2} \int_{\underline{v}_{1}}^{\bar{v}_{1}} \max \left\{0, v_{1}-K_{-1}\right\} d F_{1}\left(v_{1}\right) \\
& =R_{1}\left(K_{-1} \mid F_{1}\right),
\end{aligned}
$$

where the inequality follows because, for any fixed $K_{-1}, \max \left\{0, v_{1}-K_{-1}\right\}$ is an increasing convex function of $v_{1}$ and we assumed that either $F_{1}$ first-order stochastically dominates $\hat{F}_{1}$ or $\hat{F}_{1}$ second-order stochastically dominates $F_{1}$. Thus, changing player 1's distribution from $F_{1}$ to $\hat{F}_{1}$ (weakly) shifts player 1's best-response function leftward, as shown in Figure 5. Therefore, the equilibrium values of the expected contributions $\left(K_{1}, K_{-1}\right)$ for the profile $\left(\hat{F}_{1}, F_{2}, \ldots F_{n}\right)$ will be on the function $R_{-1}$, to the northwest of the equilibrium expected contributions for the profile $\left(F_{1}, F_{2}, \ldots F_{n}\right)$. This reasoning establishes $\hat{K}_{1}^{*} \leq K_{1}^{*}$ in part a, and it is illustrated in Figure 5. The remainder of part a follows from Proposition 4.

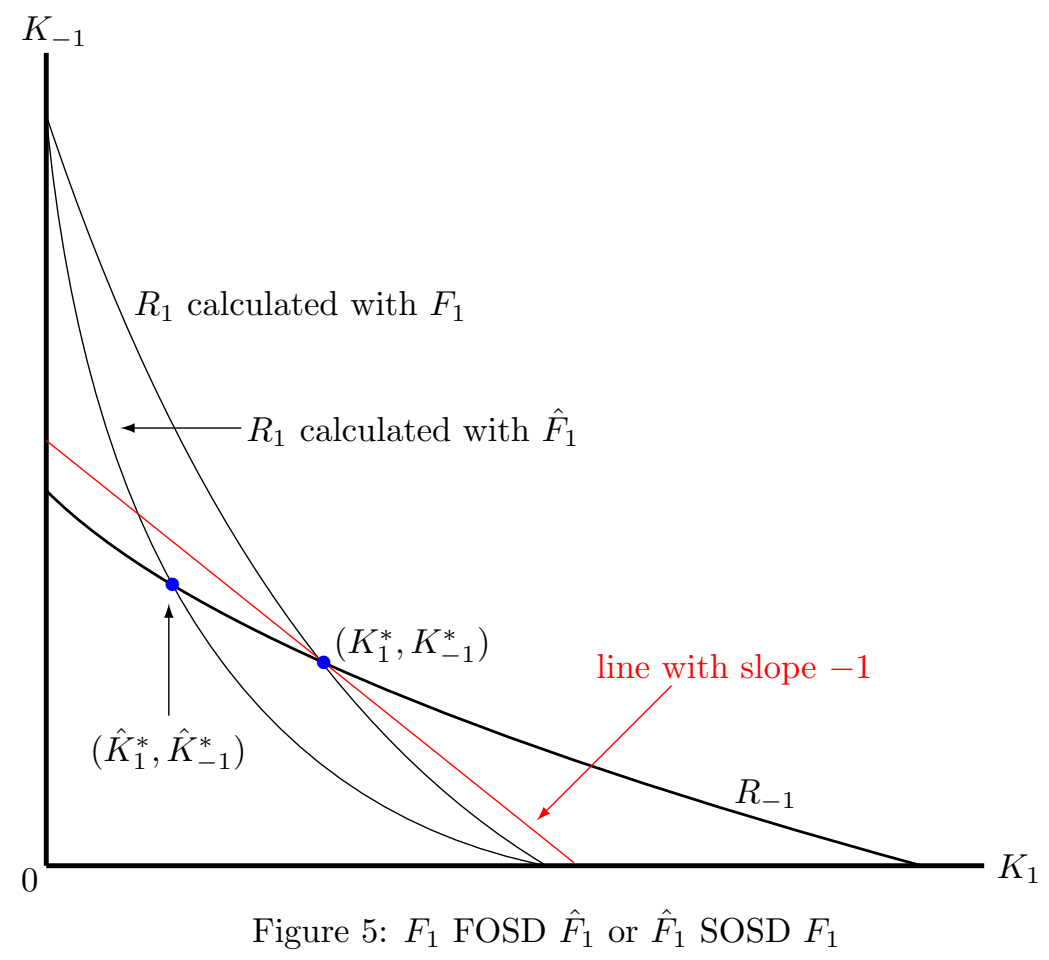

To establish part b, it is enough to consider Figure 5 again, remembering that the function $R_{-1}$ is strictly 
decreasing with slope less than 1 in absolute value (see Proposition 4 ). Therefore, the point $\left(\hat{K}_{1}^{*}, \hat{K}_{-1}^{*}\right)$ lies below the line that connects all pairs $\left(K_{1}, K_{-1}\right)$ summing to $K_{1}^{*}+K_{-1}^{*}$.

To establish part c, denote the equilibrium strategies as $s_{i}^{*}$ when the distributions are $\left(F_{1}, F_{2}, \ldots, F_{n}\right)$, and as $\hat{s}_{i}^{*}$ when the distributions are $\left(\hat{F}_{1}, F_{2}, \ldots, F_{n}\right)$. Such equilibrium strategies are described in Proposition 3 , for $\left(K_{1}^{*}, K_{2}^{*}, \ldots, K_{n}^{*}\right)$ and $\left(\hat{K}_{1}^{*}, \hat{K}_{2}^{*}, \ldots, \hat{K}_{n}^{*}\right)$, respectively. We then have

$$
U_{1}\left(\hat{s}_{1}^{*}\left(v_{1}\right) \mid v_{1}, \hat{K}_{-1}^{*}\right) \geq U_{1}\left(s_{1}^{*}\left(v_{1}\right) \mid v_{1}, \hat{K}_{-1}^{*}\right) \geq U_{1}\left(s_{1}^{*}\left(v_{1}\right) \mid v_{1}, K_{-1}^{*}\right),
$$

where the first inequality follows because $\hat{s}_{1}^{*}$ is optimal against $\hat{K}_{-1}^{*}$, and the second inequality follows because $\hat{K}_{-1}^{*} \geq K_{-1}^{*}$ and the expression for $U_{1}$ is increasing in the expected contribution of the other agents (see (13)). Note that the first term in the chain of inequalities above is the equilibrium payoff for type $v_{1}$ under $\left(\hat{F}_{1}, F_{2}, \ldots, F_{n}\right)$, while the last term in the chain of inequalities is the equilibrium payoff of type $v_{1}$ under $\left(F_{1}, F_{2}, \ldots, F_{n}\right)$, so we have established part c. To establish part d, we first note that, for any $j \geq 2$, we have $\sum K_{i}^{*}-\sum \hat{K}_{i}^{*} \geq 0 \geq K_{j}^{*}-\hat{K}_{j}^{*}$, by parts a and b, so that

$$
\sum K_{i}^{*}-K_{j}^{*} \geq \sum \hat{K}_{i}^{*}-\hat{K}_{j}^{*}
$$

proceeding as in part c, we obtain

$$
U_{j}\left(s_{j}^{*}\left(v_{j}\right) \mid v_{j}, \sum K_{i}^{*}-K_{j}^{*}\right) \geq U_{j}\left(\hat{s}_{j}^{*}\left(v_{j}\right) \mid v_{j}, \sum K_{i}^{*}-K_{j}^{*}\right) \geq U_{j}\left(\hat{s}_{j}^{*}\left(v_{j}\right) \mid v_{j}, \sum \hat{K}_{i}^{*}-\hat{K}_{j}^{*}\right),
$$

thus establishing the desired inequalities.

Part a of Proposition 5 confirms the intuition in Example $3{ }^{7}$ Part b proves that, while the expected contributions of the first agent and those of all other agents move in opposite directions, the sign of the effect on overall contributions is determined by the sign of the change for the first agent's contribution. This is because of the imperfect crowding-out result in Proposition 4. It states that all other agents' contributions react in the opposite direction of a change in the expected contribution of agent 1 , but not sufficiently to fully offset it. The intuition for part $\mathrm{c}$ is simple: when moving from distributions $\left(F_{1}, F_{2}, \ldots, F_{n}\right)$ to $\left(\hat{F}_{1}, F_{2}, \ldots, F_{n}\right)$, all agents other than agent 1 are contributing more. Therefore, agent 1's utility increases. The intutition for part $\mathrm{d}$ is similar, once one establishes that any agent $j$ different than 1 perceives that all other agents

\footnotetext{
${ }^{7}$ As the proof makes clear, what is needed for this result is convexity of equilibrium strategies. The uniform threshold assumption yields piecewise-linear equilibrium strategies. Therefore, assuming a uniform threshold, while helpful, is not essential for our result.
} 
contribute less, in aggregate. To see this last point, observe that, for $j \neq 1$,

$$
\sum_{i \neq j} \hat{K}_{i}^{*}=\sum \hat{K}_{i}^{*}-\hat{K}_{j}^{*} \leq \sum K_{i}^{*}-K_{j}^{*}=\sum_{i \neq j} K_{i}^{*}
$$

where the inequality follows from parts a and b of Proposition 5.

Proposition 5 has implications for adding another member to the group of participants. Consider Proposition 5 where according to distribution $\hat{F}_{1}$ player 1 is sure to have value 0 while for distribution $F$ player 1 's value has a nondegenerate distribution. When player 1's value is sure to be 0 , he is effectively a nonparticipant in the game. Because $F_{1}$ first-order stochastically dominates $\hat{F}_{1}$, we have the following result.

Corollary 1 (Adding a participant). If a new potential contributor is added to the group, then the expected contribution of each original member falls but total expected contributions increase. Moreover, each original member of the new group, for each possible value, is at least as well off as without the additional member.

The comparisons in Proposition 5 consider changing the distribution of values for only a single player. For symmetric games, we can also make clear comparisons when the change in the common distribution of values can be described by stochastic dominance.

Corollary 2 (Stochastic dominance in symmetric games). Consider two distributions of players' values, $F$ and $\hat{F}$. All $n$ players' values are independently and identically distributed, in the first case according to cdf $F$, and in the second according to $\hat{F}$. If either $F$ first-order stochastically dominates $\hat{F}$ or $\hat{F}$ second-order stochastically dominates $F$, then $K^{*} \geq \hat{K}^{*}$. Moreover, for each possible value, a player's payoff is at least as large when the common distribution of values is $F$ as when it is $\hat{F}$.

The first part of Corollary 2 follows from repeated application of Proposition 5.b. As the profile of players' distributions changes from $(\hat{F}, \hat{F}, \ldots, \hat{F})$ to $(F, \hat{F}, \ldots, \hat{F})$ to $\ldots$ to $(F, \ldots, F, \hat{F})$ to $(F, \ldots, F, F)$, total expected contributions weakly increase. Thus, the total expected efforts at the beginning and end of this chain of changes satisfy $n \hat{K}^{*} \leq n K^{*}$. The second part of Corollary 2 follows from (13), where it is clear that for any value $v_{1}$, for example, player 1 is at least as well off when the common distribution of values is $F$ as when it is $\hat{F}$ because all other players contribute more under $F$ than under $\hat{F}$ (i.e., $\left.(n-1) K^{*} \geq(n-1) \hat{K}^{*}\right)$.

A graphical derivation of Corollary 2's contribution ranking is also available. As explained in the proof of Proposition 5, the change in distributions from $F$ to $\hat{F}$ (weakly) shifts player 1's "best response" function $R_{1}$ leftward as depicted in Figure 6. The unique equilibrium is symmetric and satisfies the two conditions, i) $K^{*}=R_{1}\left((n-1) K_{-1}^{*}\right)$ and ii) $K_{-1}^{*}=(n-1) K^{*}$. In Figure 6 the equilibrium is found at the intersection of the reaction function (condition i)) and the symmetry line (condition ii)). For the two different distributions, Figure 6 shows $\hat{K}^{*} \leq K^{*}$. 


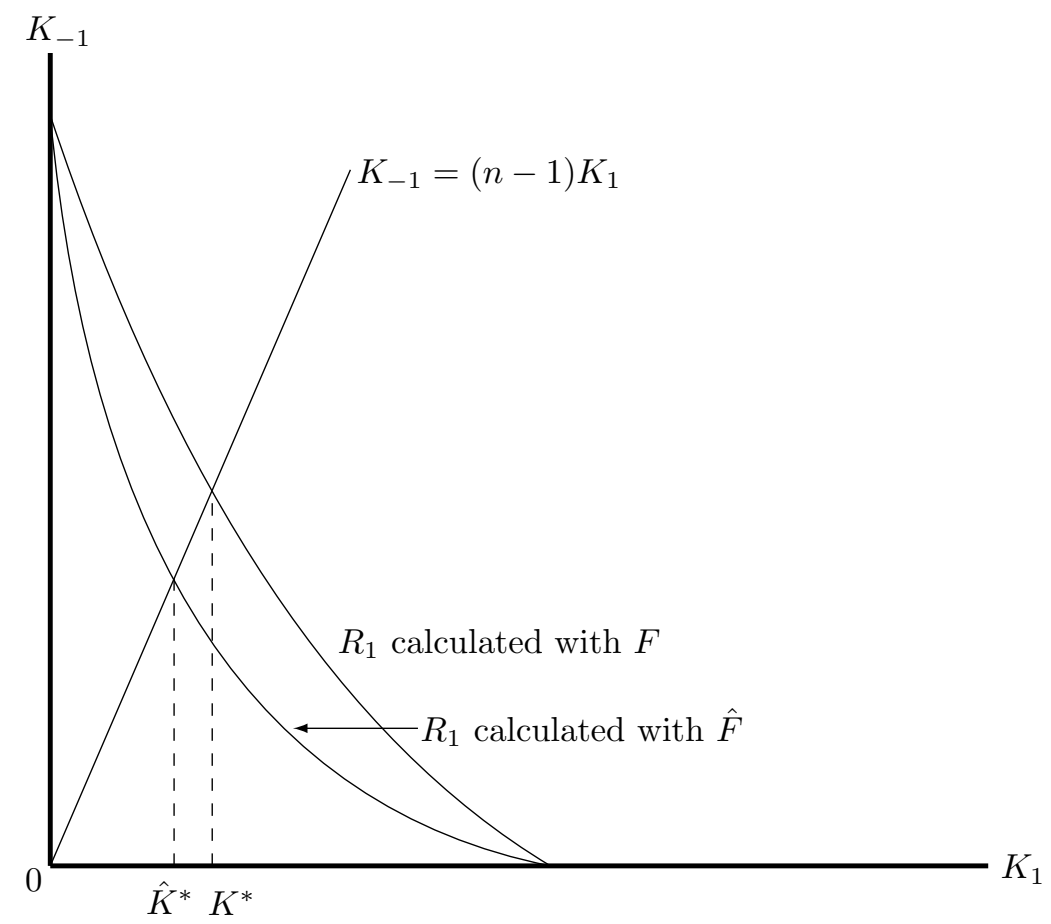

Figure 6: Symmetric equilibria when $F$ FOSD $\hat{F}$ or $\hat{F}$ SOSD $F$

It is worth pointing out that results similar to those in Proposition 5 and Corollaries 1 and 2 have not been available for the subscription game with fixed threshold, where technical difficulties make situations with asymmetries or more than two players analytically intractable. In contrast, connections with observed behavior are easier within our model. For example, increasing a player's values by first-order stochastic dominance leads that player, quite naturally, to greater expected contributions. This implication has been corroborated by the empirical literature on lobbying surveyed by Potters and Sloof (1996). ${ }^{8}$

As a particular illustration of second-order stochastic dominance in Corollary 2, consider three symmetric distributions on $[0,1]$ having mean $1 / 2$. The first is a unit mass at $1 / 2$, the second is the uniform distribution on $[0,1]$, and the third is the two-point distribution where 0 and $1 / 2$ each have probability $1 / 2$. These are ordered by second-order stochastic dominance, with the two-point distribution being the most risky and the one-point distribution the least. The expected contribution of each player is $1 / 6 \approx 0.16667$ for the one-point distribution, 0.17157 for the uniform distribution, and $1 / 5=0.2$ for the two-point distribution.

Our results for second-order stochastic dominance may contribute to the literature on the advantages or disadvantages of ethnic fragmentation in games of trust, team production, social capital, or, more generally,

\footnotetext{
${ }^{8}$ Potters and Sloof point out that "several studies indicate that economic interest groups, in particular, tend to give more money to representatives who join important committees." Even more directly, "An interest group that has a large stake in influencing policy-makers and regulatory agents is hypothesized to be more politically active and hence to have a larger impact on policy ... the stake variables ... are often significant and usually have the predicted sign." See Potters and Sloof (1996, p. 412 and pp. 416-417) and references therein.
} 
in cooperative endeavors. Various authors show the effect of increased heterogeneity may be positive or negative. This vast empirical and theoretical literature is well surveyed by Alesina and La Ferrara (2005), who identify three ways to formalize how heterogeneity affects economic outcomes: through preferences, through an influence on available strategies, and through the production function. ${ }^{9}$ These formalizations are important and insightful. However, none appears to capture a facet of interethnic relations that plays an important role in the sociology literature: ignorance about each other because of lack of social contact. ${ }^{10}$ A natural formalization of the fact that agents are more ignorant about members of other ethnic or racial groups than about members of their own group is that agents perceive "outsiders" as more risky, in terms of second-order stochastic dominance. ${ }^{11}$ Part b of Proposition 5, for the specific case in which the second-order stochastic dominance ranking applies to a minority of only one agent, yields the surprising result that, in expectation, total contributions are larger in a non-homogenous group rather than in the homogenous group. Corollary 2 may be used to extend the comparison to the other extreme case where each agent represents a different ethnic group. Intermediate and more realistic comparisons appear more complex to decipher because of the feedback effect generated by how the minority may view the values of the majority, and a full analysis is beyond the scope of this paper. Nonetheless, we believe that ignorance may be usefully added to the three-way classification in Alesina and La Ferrara (2005), and its effects can be explored along the lines of Proposition 5.

Proposition 5 and Corollary 2 have important normative implications as well: they point at free-riding as the major sources of inefficient underprovision in our framework, rather than asymmetric information. In fact, total contributions are larger when agents are uncertain about each other's values. Moreover, in the presence of private information a weaker definition of efficiency is appropriate. This issue is explored in the next section.

\section{Interim Incentive Efficiency}

The main objective of this section is to determine whether the equilibrium allocation in Proposition 3 is efficient. We maintain the assumption that the threshold cost is uniformly distributed over $[0, \bar{c}]$. We first clarify the notion of efficiency we are considering, among the alternatives in the literature for environments with private information. We then apply the existing characterization results to the equilibrium allocation in Section 3, establishing that this allocation is not interim incentive efficient.

\footnotetext{
${ }^{9}$ See Alesina and La Ferrara (2005, pp. 764-766) and references therein.

${ }^{10}$ The so-called Contact Theory "...holds that the sharp rupture between the social lives of whites and blacks promotes whites' ignorance about blacks. This ignorance feeds erroneous, oversimplified, negative beliefs about blacks, which in turn engender feelings of hostility and discriminatory social and political predispositions toward blacks." See Jackman and Crane (1986, pp. 460-461) and references therein. Negative beliefs, usually interpreted as prejudice, may be formalized as agents' preference to interact with other agents who are similar to themselves, as in Alesina and La Ferrara (2000). Beyond prejudice, ignorance plays an important role in Contact Theory, and this is the aspect we focus on.

${ }^{11}$ For example, one may postulate the value of agent $i$ to be the sum of two random variables, with the realization of one of them being apparent to all other agents that share the same ethnic background of agent $i$, while the realization remains unknown to all other agents.
} 


\subsection{General Considerations}

The notion of efficiency we use is interim incentive efficiency, defined by Holmström and Myerson (1983), and further characterized by Ledyard and Palfrey (1999 and 2007). This is the same notion used by Laussel and Palfrey (2003) and Barbieri and Malueg (2008) for the subscription game with known threshold. Before proceeding further with the welfare analysis, as in many models of threshold provision of a public good, the issue arises of how, for efficiency considerations, to deal with contributions in excess of the threshold. Here we follow Nitzan and Romano (1991), Laussel and Palfrey (2003), and Barbieri and Malueg (2008) in assuming that excess contributions are not wasted, but they accrue to the producer of the public good. We adopt the formalization of Laussel and Palfrey (2003), who introduce a new agent, the collector, in addition to the $n$ players described in Section 2, whom we refer to as contributors in this section. The collector is the only agent that can produce the public good, incurring cost $c$. The collector is risk-neutral and is privately informed about $c$. With this in mind, we can reinterpret our subscription game in Section 2 as a take-it-orleave-it offer that contributors make to the collector. Each contributor commits to paying some amount only if the collector provides the public good, and zero otherwise. After seeing the sum of promised payments, and taking into consideration the realization of the cost of production $c$, the collector decides whether to accept the contributors' offer. If the offer is rejected, the public good is not produced and all players receive zero payoffs. If the contributors' offer is accepted, the public good is produced, the payoff of the collector is the sum of contributors' offers minus $c$, and the payoff of each contributor $i$ is $v_{i}$ - (player $i$ 's contribution), just as in Section 2. In the subgame perfect equilibrium, the collector accepts those and only those offers that sum to at least $c$. Therefore, the contributors' problem is equivalent to the one solved in Sections 2 and 3.

It is now possible to describe an interim incentive efficient equilibrium allocation. We consider each type of the collector and each type of each contributor as separate individuals. The equilibrium allocation deriving from Proposition 3 and from the optimal behavior of the collector described above is interim incentive efficient if it is not Pareto dominated by another allocation resulting from any incentive compatible, and individually rational mechanism. Note how this definition of efficiency takes into account the limitations that private information imposes on the social planner trying to implement alternative allocations. The definition of individually rational we use is what Ledyard and Palfrey (2007) call "standard": the utility of the outside option is constant in type and equal to zero.

Any discussion of interim incentive efficiency requires a discussion of mechanism design. By the Revelation Principle it suffices to consider only direct mechanisms that are feasible, that is, both incentive compatible and individually rational. Direct mechanisms are pairs of functions $(p, x)$ defined on $[\underline{v}, \bar{v}] \times[0, \bar{c}]$ (where $\left.[\underline{v}, \bar{v}] \equiv\left[\underline{v}_{1}, \bar{v}_{1}\right] \times \cdots \times\left[\underline{v}_{n}, \bar{v}_{n}\right]\right)$. Each player $i$ reports value $v_{i}^{\prime}$ and the collector reports $c^{\prime} ;$ then, denoting 
with $v^{\prime}$ the vector $\left(v_{1}^{\prime}, \ldots, v_{n}^{\prime}\right), x_{i}\left(v^{\prime}, c^{\prime}\right)$ is player $i$ 's payment to the collector and $p\left(v^{\prime}, c^{\prime}\right) \in[0,1]$ is the probability the good is provided. Under mechanism $(p, x)$, when others report truthfully, player $i$ with value $v_{i}$ reporting $v_{i}^{\prime}$ obtains payoff

$$
\hat{U}_{i}\left(v_{i}^{\prime} \mid v_{i}\right) \equiv \int_{0}^{\bar{c}} \int_{\underline{v}_{-i}}^{\bar{v}_{-i}}\left(v_{i} p\left(v_{-i}, v_{i}^{\prime}, c\right)-x_{i}\left(v_{-i}, v_{i}^{\prime}, c\right)\right) d F_{-i}\left(v_{-i}\right) d H(c)
$$

and the collector with cost $c$ reporting $c^{\prime}$ obtains payoff

$$
\hat{U}_{c}\left(c^{\prime} \mid c\right) \equiv \int_{\underline{v}}^{\bar{v}}\left(\sum x_{i}\left(v, c^{\prime}\right)-c p\left(v, c^{\prime}\right)\right) d F(v)
$$

Define $U_{i}^{*}\left(v_{i}\right) \equiv \hat{U}_{i}\left(v_{i} \mid v_{i}\right), i=1, \ldots, n$, and $U_{c}^{*}(c) \equiv \hat{U}_{c}(c \mid c)$ as the associated truth-telling payoffs. The Envelope Theorem then implies that, for an incentive compatible mechanism $(p, x)$,

$$
\frac{d U_{i}^{*}\left(v_{i}\right)}{d v_{i}}=\int_{0}^{\bar{c}} \int_{\underline{v}_{-i}}^{\bar{v}_{-i}} p\left(v_{-i}, v_{i}^{\prime}, c\right) d F_{-i}\left(v_{-i}\right) d H(c)
$$

and

$$
\frac{d U_{c}^{*}(c)}{d c}=-\int_{\underline{v}}^{\bar{v}} p\left(v, c^{\prime}\right) d F(v)
$$

integration of these two formulas gives payoffs under an incentive compatible mechanism:

$$
U_{i}^{*}\left(v_{i}\right)=U_{i}^{*}\left(\underline{v}_{i}\right)+\int_{\underline{v}_{i}}^{v_{i}} \int_{0}^{\bar{c}} \int_{\underline{v}_{-i}}^{\bar{v}_{-i}} p\left(v_{-i}, s, c\right) d F_{-i}\left(v_{-i}\right) d H(c) d F_{i}(s), \quad i=1, \ldots, n
$$

and, for the collector,

$$
U_{c}^{*}(c)=U_{c}^{*}(\bar{c})+\int_{c}^{\bar{c}} \int_{\underline{v}}^{\bar{v}} p(v, s) d F(v) d H(s) .
$$

Before we address the interim efficiency of the subscription game equilibrium derived above, we provide a fairly general sufficient condition for inefficiency of a mechanism in this public good environment. The value of this sufficient condition is that it does not require the monotonicity of virtual valuations, whether typeweighted or not, thus covering situations beyond the "regular" case defined in Ledyard and Palfrey (2007). It is convenient first to define the sum of virtual valuations $S(v, c)$ as follows: ${ }^{12}$

$$
S(v, c) \equiv \sum\left(v_{i}-\frac{1-F_{i}\left(v_{i}\right)}{f_{i}\left(v_{i}\right)}\right)-\left(c+\frac{H(c)}{h(c)}\right)
$$

\footnotetext{
${ }^{12}$ The virtual valuation for the seller is $-\left(c+\frac{H(c)}{h(c)}\right)$, which becomes $-2 c$ when $H$ is the uniform distribution on $[0, \bar{c}]$.
} 
Proposition 6 (A sufficient condition for inefficiency). Let $(p, x)$ be an incentive compatible, individually rational direct mechanism. Suppose there exists an allocation rule $\tilde{p}(v, c)$ such that the following conditions are satisfied:

1. $\forall c, \int_{\underline{v}}^{\bar{v}} \tilde{p}(v, c) d F(v) \geq \int_{\underline{v}}^{\bar{v}} p(v, c) d F(v)$ and

$$
\int_{0}^{\bar{c}} \int_{\underline{v}_{-i}}^{\overline{v_{-i}}} \tilde{p}(v, c) d F_{-i}\left(v_{-i}\right) d H(c) \geq \int_{0}^{\bar{c}} \int_{\underline{v}_{-i}}^{\bar{v}_{-i}} p(v, c) d F_{-i}\left(v_{-i}\right) d H(c), \forall v_{i}, i
$$

2. $\forall i, \int_{0}^{\bar{c}} \int_{\underline{v}_{-i}}^{\bar{v}} \tilde{p}(v, c) d F_{-i}\left(v_{-i}\right) d H(c)$ is increasing in $v_{i}$ and $\int_{\underline{v}}^{\bar{v}} \tilde{p}(v, c) d F(v)$ is decreasing in $c$; and

3. $\varepsilon \equiv \int_{0}^{\bar{c}} \int_{\underline{v}}^{\bar{v}} S(v, c)(\tilde{p}(v, c)-p(v, c)) d F(v) d H(c)>0$.

Then $(p, x)$ is not interim incentive efficient.

Proof. Condition 1 states that the interim probability of completion for each type is at least as large under $\tilde{p}$ as under $p$, while Condition 2 is the usual second-order condition for incentive compatibility of $\tilde{p}$. Condition 3 states that the overall surplus, under incentive compatibility, is larger for $\tilde{p}$ than for $p$. Because $(p, x)$ is incentive compatible and individually rational, the "only if" implication Lemma 3 in Ledyard and Palfrey (2007) then yields

$$
\sum U_{i}^{*}\left(\underline{v}_{i}\right)+U_{c}^{*}(\bar{c})=\int_{0}^{\bar{c}} \int_{\underline{v}}^{\bar{v}} S(v, c) p(v, c) d F(v) d H(c)
$$

so that

$$
\begin{aligned}
\int_{0}^{\bar{c}} \int_{\underline{v}}^{\bar{v}} S(v, c) & \tilde{p}(v, c) d F(v) d H(c)-\left[\sum U_{i}^{*}\left(\underline{v}_{i}\right)+\left(U_{c}^{*}(\bar{c})+\varepsilon\right)\right] \\
& =\int_{0}^{\bar{c}} \int_{\underline{v}}^{\bar{v}} S(v, c) \tilde{p}(v, c) d F(v) d H(c)-\int_{0}^{\bar{c}} \int_{\underline{v}}^{\bar{v}} S(v, c) \tilde{p}(v, c) d F(v) d H(c)-\varepsilon \\
& =\int_{0}^{\bar{c}} \int_{\underline{v}}^{\bar{v}} S(v, c)(\tilde{p}(v, c)-p(v, c)) d F(v) d H(c)-\varepsilon \\
& =0
\end{aligned}
$$

where the last equality follows from Condition 3. Therefore, the "if" implication of Lemma 3 in Ledyard and Palfrey (2007), along with Condition 2, imply that, using allocation rule $\tilde{p}$, an incentive compatible and individually rational mechanism exists in which the utility of the lowest type of each player is the same as for $p$, and in which the utility of the worst type for the seller is strictly higher, in particular it is increased by $\varepsilon>0$. To conclude the proof, note that Condition 1 and the usual incentive compatibility lemma (Lemma 1 
in Ledyard and Palfrey (2007)) imply each type of the buyer is not hurt by the change in mechanisms, and each type of the seller is strictly better off. ${ }^{13}$ This establishes that $p$ is Pareto-dominated by $\tilde{p}$.

The subscription game is an incentive compatible, individually rational (non-direct) mechanism; for any equilibrium $\left(s_{1}, \ldots, s_{n}\right)$, using the optimal behavior of the collector described above, the equilibrium allocation rule is the following:

$$
p^{\mathrm{eq}}(v, c)= \begin{cases}1 & \text { if } \sum s_{i}\left(v_{i}\right) \geq c \\ 0 & \text { if } \sum s_{i}\left(v_{i}\right)<c .\end{cases}
$$

We now consider the case of the uniformly distributed threshold, so a unique equilibrium exists and it characterized in Proposition 3. Next, we apply the previous proposition and construct an alternative allocation rule $p^{\text {alt }}$ that deviates from $p^{\text {eq }}$ where $p^{\text {eq }}=0$ and $S(v, c)>0$. For a subset of this parameter region, we set $p^{\text {alt }}=1$, being careful that Condition 2 in Proposition 6 is satisfied. ${ }^{14}$ We thus obtain the following.

Proposition 7 (Inefficiency). The subscription game with a uniformly distributed uncertain threshold is not interim incentive efficient.

Proof. Without loss of generality, we may assume for any $i$ that $s_{i}\left(\bar{v}_{i}\right)>0$; otherwise, the contributor is always inactive and can be disregarded in the analysis below. Define $\hat{c} \equiv\left(\sum \bar{v}_{i}-\sum K_{i}\right) / 2$. Note that, in equilibrium, the probability of completion is zero for any $c \geq \hat{c}$. At the same time, we have $S(\bar{v}, \hat{c})=\sum K_{i}>$ 0 . Moreover, we choose $\eta>0$ sufficiently small that for any $c>\hat{c}-\eta$, if $\sum s_{i}\left(v_{i}\right)>c$ then $s_{i}\left(v_{i}\right)>0$ for all $i$ (this is ensured by taking $\eta<\min _{j} s_{j}\left(\bar{v}_{j}\right)$ ). We may also choose $\eta>0$ sufficiently small so that $\hat{c}+\eta<\bar{c}$ and, by continuity of $S$, for any $c \in(\hat{c}-\eta, \hat{c}+\eta)$ and for any profile of values such that $\sum v_{i} \in\left(\sum \bar{v}_{i}-2 \eta, \sum \bar{v}_{i}\right)$, we have $S(v, c)>0$.

Observe that if $c>\hat{c}-\eta$, then the good is provided only if all contributions are strictly positive; therefore, on this restricted region (20) becomes the following:

$$
p^{\mathrm{eq}}(v, c)= \begin{cases}1 & \text { if } \sum v_{i} \geq g^{\mathrm{eq}}(c) \equiv 2 c+\sum K_{i} \\ 0 & \text { if } \sum v_{i}<g^{\mathrm{eq}}(c) .\end{cases}
$$

We now construct the alternative provision function $p^{\text {alt }}(v, c)$, first defining the function $g^{\text {alt }}$. For $c \leq \hat{c}-\eta$, define $g^{\text {alt }}(c)=g^{\text {eq }}(c)$; for $c>\hat{c}-\eta$, define $g^{\text {alt }}(c) \equiv g^{\text {eq }}(\hat{c}-\eta)+(c-(\hat{c}-\eta))$. Note these properties of $g^{\text {alt }}$ :

i. $g^{\text {alt }}(c) \leq g^{\mathrm{eq}}(c)$, for any $c \geq \hat{c}-\eta$, with equality only at $c=\hat{c}-\eta$;

ii. $g^{\text {alt }}$ is increasing in $c$;

\footnotetext{
${ }^{13}$ As usual, $\varepsilon>0$ may alternatively be distributed among all players, to make every type of each player better off.

${ }^{14} \mathrm{~A}$ similar strategy of proof for the 2-person double auction appears in Satterthwaite and Williams (1989).
} 
iii. $g^{\text {alt }}(\hat{c}+\eta)=g^{\mathrm{eq}}(\hat{c}-\eta)+2 \eta=\sum \bar{v}_{i}$.

These properties are illustrated in Figure 7, which depicts the functions $g^{\text {eq }}$ and $g^{\text {alt }}$ (note that the origin depicted assumes a strictly positive value of $\left.\sum v_{i}\right)$. We now define $p^{\text {alt }}$ as follows:

$$
p^{\text {alt }}(v, c)= \begin{cases}1 & \text { if } \sum v_{i} \geq g^{\text {alt }}(c) \\ 0 & \text { if } \sum v_{i}<g^{\text {alt }}(c)\end{cases}
$$

Figure 7 also depicts the relationship between $p^{\text {eq }}$ and $p^{\text {alt }}$ (the solid lines bound the regions where values of $p^{\text {eq }}$ and $p^{\text {alt }}$ are as specified).

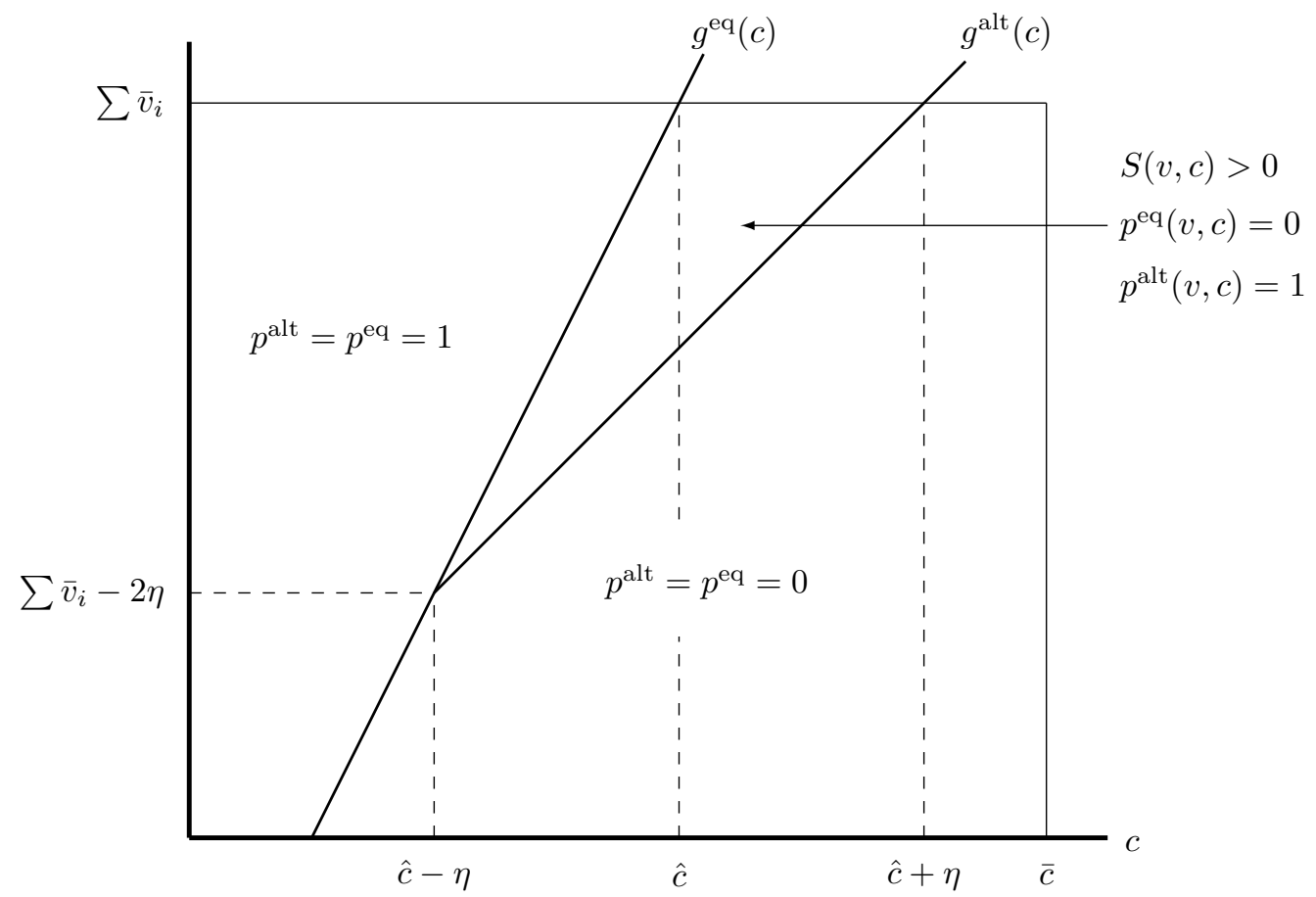

Figure 7: Construction of a Pareto superior allocation function, $p^{\text {alt }}$

To complete the proof, we now proceed to verify all conditions of Proposition 6 . Condition 1 follows because, using Property i, we have $p^{\mathrm{eq}}(v, c) \geq p^{\text {alt }}(v, c)$. Condition 2 follows because both $p^{\mathrm{eq}}(v, c)$ and $p^{\text {alt }}(v, c)$ are increasing in each $v_{i}$ and decreasing in $c$ ( the latter because both $g^{\text {eq }}$ and $g^{\text {alt }}$ are increasing in $c$ (see Property ii above)). Moreover, by Property iii, the expected probability of completion for the seller under $p^{\text {alt }}$ is continuous at $c=\hat{c}+\eta$, where it attains the value of zero. Condition 3 follows because, given Property i, there exist a full-measure set such that $p^{\text {alt }}(v, c)>p^{\mathrm{eq}}(v, c)$, and, on that full-measure set, $S(v, c)>0$, as the continuity argument above establishes. 


\section{Conclusion}

This paper has picked up where Nitzan and Romano (1990) left off, calling for their model with threshold uncertainty to be extended to allow for private values among contributors. ${ }^{15}$ Alternatively, one may view our paper as introducing threshold uncertainty into the private-information subscription game. We have shown this approach combines the best features of both strands of the literature: our framework adds the sharpness of prediction of Nitzan and Romano's model to the interest for applications that models of private information entail, a potential heretofore unexplored because of technical difficulties. Therefore, the primary interest in our results, beyond confirming those Nitzan and Romano (1990), lies in the comparison with models having private values and certain threshold. In particular, adding threshold uncertainty yields much sharper positive predictions and normative conclusions.

We have established under very general conditions that the introduction of private information into Nitzan and Romano's model is still consistent with existence of (a Bayesian) equilibrium, and if the distribution of threshold uncertainty is concave with support $[0, \bar{c}]$, then the equilibrium is unique. After establishing these existence and uniqueness results, our analysis focused on the case where the threshold cost is uniformly distributed over $[0, \bar{c}]$. Admittedly, this particular introduction of cost uncertainty is special, but we were more interested in comparative statics regarding the distributions of players' values, ${ }^{16}$ and this approach paid big dividends in that equilibrium was easily characterized and seen to be unique, regardless of the number of players or their distributions. ${ }^{17}$ This allowed us to analyze player-specific changes both in intensity and dispersion of values. Nitzan and Romano's analysis implies that if the highest possible value of the cost exceeds the sum of all players' values, then any equilibrium is classically inefficient-there will be realizations of cost where it is socially desirable to provide the good but contributions fall short. With private information about values, interim incentive efficiency is the appropriate measure of efficiency. Our analysis extends Nitzan and Romano's negative result: we show that if the maximum possible cost, $\bar{c}$, is at least as large as the largest possible sum of players' values for the good, $\sum \bar{v}_{i}$, then the equilibrium is interim incentive inefficient.

Future work may proceed in two directions. First there is the technical challenge of incorporating cost distributions other than the uniform distribution on $[0, \bar{c}]$. Our results for the two-player case are encouraging, because uniqueness is preserved when $H$ is concave. However, more work is needed to completely describe the $n$-player case. One might also consider uniform distributions with support bounded away from 0. Such distributions raise the possibility that for some realizations of a player's value, it is certain the good will not

\footnotetext{
${ }^{15}$ See Nitzan and Romano (1990, p. 369).

${ }^{16}$ This is in contrast to McBride (2006), who focused on varying the distribution of cost while having for all players a common value of the public good.

${ }^{17}$ Players' distributions were only assumed to be independent, continuous, and with bounded support.
} 
be provided (see Alboth et al., 2001, and Barbieri and Malueg, 2008), which introduces some multiplicity of equilibria. A second area for research would fully investigate how differences among individuals or groups might hinder or facilitate interactions. Our framework offers a laboratory for studying such effects where interactions with less familiar groups could be modeled through the relative dispersion in perceived values of members of own versus different groups. 


\section{Appendix}

Proof of Proposition 1. We verify, in order, that the conditions of Corollary 2.1 in Athey (2001) are satisfied.

First, Athey's measurability condition A1 is satisfied in our framework. She also requires densities of players' values to be atomless and bounded, which we have assumed. Her assumption that a player's expected payoff, conditional on value being in a convex set, exist and be finite is satisfied because the $\left(v_{i}-x_{i}\right) H\left(\sum s_{j}\left(v_{j}\right)\right)$ is bounded and integrable for all nondecreasing functions $s_{j}, j \neq i$.

Next, her condition i) requires that we can restrict a player's contributions to a compact interval. Any contribution exceeding $\bar{c}$ is strictly dominated by a contribution of $\bar{c}$. Therefore, we may restrict each player's choice of $x$ to the interval $[0, \bar{c}]$, so condition i) is satisfied. Athey's condition ii) requires the integrand $\left(v_{i}-x_{i}\right) H\left(\sum x_{j}\right)$ to be continuous in $\left(x_{1}, \ldots, x_{n}\right)$. This is satisfied because we assumed $H$ is continuous.

It only remains to check that Athey's single-crossing condition (SCC) is satisfied by $U_{i}\left(x \mid v_{i}\right)$ for any choice of nondecreasing strategies for players other than $i$. Let such nondecreasing strategies $\left(s_{j}\right)_{j \neq i}$ be given. Athey's SCC is stated as follows: for any $x_{H}>x_{L}$ and $v_{H}>v_{L}$,

$$
U_{i}\left(x_{H} \mid v_{L}\right)-U_{i}\left(x_{L} \mid v_{L}\right) \geq 0 \Longrightarrow U_{i}\left(x_{H} \mid v_{H}\right)-U_{i}\left(x_{L} \mid v_{H}\right) \geq 0
$$

and

$$
U_{i}\left(x_{H} \mid v_{L}\right)-U_{i}\left(x_{L} \mid v_{L}\right)>0 \Longrightarrow U_{i}\left(x_{H} \mid v_{H}\right)-U_{i}\left(x_{L} \mid v_{H}\right)>0 .
$$

For the remainder of the proof, suppose $x_{H}>x_{L}$ and $v_{H}>v_{L}$. From the definition of $U_{i}$ we have

$$
\begin{aligned}
U_{i}\left(x_{H} \mid v_{H}\right)-U_{i}\left(x_{L} \mid v_{H}\right) & \geq(>) U_{i}\left(x_{H} \mid v_{L}\right)-U_{i}\left(x_{L} \mid v_{L}\right) \\
& \Longleftrightarrow U_{i}\left(x_{H} \mid v_{H}\right)-U_{i}\left(x_{H} \mid v_{L}\right) \geq(>) U_{i}\left(x_{L} \mid v_{H}\right)-U_{i}\left(x_{L} \mid v_{L}\right) \\
& \Longleftrightarrow \operatorname{Pr}\left(x_{H}+\sum_{j \neq i} s_{j}\left(v_{j}\right) \geq c\right) \geq(>) \operatorname{Pr}\left(x_{L}+\sum_{j \neq i} s_{j}\left(v_{j}\right) \geq c\right) .
\end{aligned}
$$

Because $x_{H}>x_{L}$, the weak inequality in (25) is satisfied, implying that (22) is satisfied. Finally, to show that (23) is satisfied, it suffices to show that if $U_{i}\left(x_{H} \mid v_{L}\right)-U_{i}\left(x_{L} \mid v_{L}\right)>0$, then the strict inequality in (25) holds, implying by (24) that (23) holds. We first show that

$$
U_{i}\left(x_{H} \mid v_{L}\right)-U_{i}\left(x_{L} \mid v_{L}\right)>0 \Longrightarrow x_{L}<v_{L}
$$


If this is not the case, then

$$
\begin{aligned}
0 & <U_{i}\left(x_{H} \mid v_{L}\right)-U_{i}\left(x_{L} \mid v_{L}\right) \\
& =\left(v_{L}-x_{H}\right) \operatorname{Pr}\left(x_{H}+\sum_{j \neq i} s_{j}\left(v_{j}\right) \geq c\right)-\left(v_{L}-x_{L}\right) \operatorname{Pr}\left(x_{L}+\sum_{j \neq i} s_{j}\left(v_{j}\right) \geq c\right) \\
& \leq\left[\left(v_{L}-x_{H}\right)-\left(v_{L}-x_{L}\right)\right] \operatorname{Pr}\left(x_{H}+\sum_{j \neq i} s_{j}\left(v_{j}\right) \geq c\right) \\
& =-\left(x_{H}-x_{L}\right) \operatorname{Pr}\left(x_{H}+\sum_{j \neq i} s_{j}\left(v_{j}\right) \geq c\right)
\end{aligned}
$$

this strict inequality is impossible because $x_{H}>x_{L}$ and probabilities are nonnegative. This contradiction implies $x_{L}<v_{L}$. We can now further conclude that

$$
U_{i}\left(x_{H} \mid v_{L}\right)-U_{i}\left(x_{L} \mid v_{L}\right)>0 \Longrightarrow x_{H}<v_{L}
$$

This must surely be the case because by (26) we know $U_{i}\left(x_{L} \mid v_{L}\right) \geq 0$; therefore the strict inequality in (27) implies the first term on the right-hand side of (27) must be strictly positive, implying $v_{L}-x_{H}>0$. Rearranging the extremes of (27) and (28) now yields

$$
\operatorname{Pr}\left(x_{H}+\sum_{j \neq i} s_{j}\left(v_{j}\right) \geq c\right)>\left(\frac{v_{L}-x_{L}}{v_{L}-x_{H}}\right) \operatorname{Pr}\left(x_{L}+\sum_{j \neq i} s_{j}\left(v_{j}\right) \geq c\right) \geq \operatorname{Pr}\left(x_{L}+\sum_{j \neq i} s_{j}\left(v_{j}\right) \geq c\right)
$$

which establishes the strict inequality in (25).

Proof of Lemma 1. The first step in determining $O_{1}\left(\tilde{s}_{1}+d\right)\left(v_{1}\right)$ is calculating $T_{2}\left(\tilde{s}_{1}+d\right)\left(v_{1}\right)$. This is accomplished using equation (5), that is

$$
T_{2}\left(\tilde{s}_{1}+d\right)\left(v_{2}\right)=\max \left\{0, v_{2}-r_{2}\left(T_{2}\left(\tilde{s}_{1}+d\right)\left(v_{2}\right), \tilde{s}_{1}+d\right)\right\}
$$

as long as all terms on the right-hand side are well-defined for $T_{2}\left(\tilde{s}_{1}+d\right)\left(v_{2}\right) \in\left[0, v_{2}\right]$. The discussion preceding the statement of Proposition 2 implies that for any $v_{1}, 0 \leq \tilde{s}_{1}\left(v_{1}\right)+d \leq \bar{v}_{1}+\underline{v}_{1}$, so that each argument of the function $h$ at the denominator in $r_{2}$ is in between 0 and $\bar{c}$, because we have assumed $\bar{c} \geq \bar{v}_{1}+\bar{v}_{2}+\max \left\{\underline{v}_{1}, \underline{v}_{2}\right\}$. Therefore, $r_{2}$ on the right-hand side of (31) is well-defined, strictly increasing in its first argument because $H$ is concave, and continuous in its first argument. This implies a solution to $(31)$ exists, it is unique, and it is in $\left[0, v_{2}\right]$ for any $v_{2}$. This verifies $T_{2}\left(\tilde{s}_{1}+d\right)$ is well-defined. Note 
that $T_{2}\left(s_{1}\right)\left(v_{2}\right)$ is well-defined by the assumption that $s_{1}$ is part of an equilibrium. We can now show $T_{2}\left(\tilde{s}_{1}+d\right)\left(v_{2}\right) \leq T_{2}\left(s_{1}\right)\left(v_{2}\right)$. Clearly, the statement is true if $T_{2}\left(\tilde{s}_{1}+d\right)\left(v_{2}\right)=0$, so by way of contradiction assume $T_{2}\left(\tilde{s}_{1}+d\right)\left(v_{2}\right)>T_{2}\left(s_{1}\right)\left(v_{2}\right)$ and $T_{2}\left(\tilde{s}_{1}+d\right)\left(v_{2}\right)>0$ for some $v_{2}$. We then have

$$
\begin{aligned}
T_{2}\left(s_{1}\right)\left(v_{2}\right) & <T_{2}\left(\tilde{s}_{1}+d\right)\left(v_{2}\right) & & \text { (by the contradiction hypothesis) } \\
& =v_{2}-r_{2}\left(T_{2}\left(\tilde{s}_{1}+d\right)\left(v_{2}\right), \tilde{s}_{1}+d\right) & & \left(\text { by } T_{2}\left(\tilde{s}_{1}+d\right)\left(v_{2}\right)>0\right) \\
& \leq v_{2}-r_{2}\left(T_{2}\left(s_{1}\right)\left(v_{2}\right), \tilde{s}_{1}+d\right) & & \text { (by the contradiction hypothesis) } \\
& \leq v_{2}-r_{2}\left(T_{2}\left(s_{1}\right)\left(v_{2}\right), s_{1}\right) & & \text { (by } \left.s_{1}<\tilde{s}_{1}+d\right) \\
& \leq T_{2}\left(s_{1}\right)\left(v_{2}\right), & & \text { (by definition in }(5))
\end{aligned}
$$

thus obtaining the contradiction $T_{2}\left(s_{1}\right)\left(v_{2}\right)<T_{2}\left(s_{1}\right)\left(v_{2}\right)$. This concludes the proof of

$$
T_{2}\left(\tilde{s}_{1}+d\right)\left(v_{2}\right) \leq T_{2}\left(s_{1}\right)\left(v_{2}\right)
$$

which we now use to show

$$
\left.O_{1}\left(\tilde{s}_{1}+d\right)\right)\left(v_{1}\right) \equiv T_{1}\left(T_{2}\left(\tilde{s}_{1}+d\right)\right)\left(v_{1}\right) \geq T_{1}\left(T_{2}\left(s_{1}\right)\right)\left(v_{1}\right) \equiv O_{1}\left(s_{1}\right)\left(v_{1}\right)
$$

and finish the proof of the lemma. Verification that $T_{1}$ is well-defined in the previous relation follows as above. Moreover, the previous relation is automatically true when $T_{1}\left(T_{2}\left(s_{1}\right)\right)\left(v_{1}\right)=0$. Therefore, by way of contradiction, suppose that for some $v_{1}$ we have $T_{1}\left(T_{2}\left(s_{1}\right)\right)\left(v_{1}\right)>T_{1}\left(T_{2}\left(\tilde{s}_{1}+d\right)\right)\left(v_{1}\right)$ and $T_{1}\left(T_{2}\left(s_{1}\right)\right)\left(v_{1}\right)>0$. We then have

$$
\begin{aligned}
T_{1}\left(T_{2}\left(\tilde{s}_{1}+d\right)\right)\left(v_{1}\right) & <T_{1}\left(T_{2}\left(s_{1}\right)\right)\left(v_{1}\right) & & \text { (by the contradiction hypothesis) } \\
& =v_{1}-r_{1}\left(T_{1}\left(T_{2}\left(s_{1}\right)\right)\left(v_{1}\right), T_{2}\left(s_{1}\right)\right) & & \left(\text { by } T_{1}\left(T_{2}\left(s_{1}\right)\right)\left(v_{1}\right)>0\right) \\
& \leq v_{1}-r_{1}\left(T_{1}\left(T_{2}\left(\tilde{s}_{1}+d\right)\right)\left(v_{1}\right), T_{2}\left(s_{1}\right)\right) & & \text { (by the contradiction hypothesis) } \\
& \leq v_{1}-r_{1}\left(T_{1}\left(T_{2}\left(\tilde{s}_{1}+d\right)\right)\left(v_{1}\right), T_{2}\left(\tilde{s}_{1}+d\right)\right) & & \text { (by }(32)) \\
& \leq T_{1}\left(T_{2}\left(\tilde{s}_{1}+d\right)\right)\left(v_{1}\right), & & \text { (by definition in }(5))
\end{aligned}
$$

thus obtaining a contradiction. This concludes the proof of the lemma establishing monotonicity.

Proof of Lemma 2. The first step is showing equation (11), that is $T_{2}\left(\tilde{s}_{1}+d\right)\left(v_{2}\right)+d>T_{2}\left(\tilde{s}_{1}\right)\left(v_{2}\right)$. We can establish all terms involved are well-defined along the lines of the proof of Lemma 1 . Clearly, the above 
statement is true if $T_{2}\left(\tilde{s}_{1}\right)\left(v_{2}\right)=0$, so by way of contradiction assume $T_{2}\left(\tilde{s}_{1}+d\right)\left(v_{2}\right)+d \leq T_{2}\left(\tilde{s}_{1}\right)\left(v_{2}\right)$ and $T_{2}\left(\tilde{s}_{1}\right)\left(v_{2}\right)>0$ for some $v_{2}$. We then have

$$
\begin{aligned}
T_{2}\left(\tilde{s}_{1}+d\right)\left(v_{2}\right)+d & \leq T_{2}\left(\tilde{s}_{1}\right)\left(v_{2}\right) & & \text { (by the contradiction hypothesis) } \\
& =v_{2}-r_{2}\left(T_{2}\left(\tilde{s}_{1}\right)\left(v_{2}\right), \tilde{s}_{1}\right) & & \left(\text { by } T_{2}\left(\tilde{s}_{1}\right)\left(v_{2}\right)>0\right) \\
& =v_{2}-r_{2}\left(T_{2}\left(\tilde{s}_{1}\right)\left(v_{2}\right)-d, \tilde{s}_{1}+d\right) & & \text { (by definition of } \left.r_{2}\right) \\
& \leq v_{2}-r_{2}\left(T_{2}\left(\tilde{s}_{1}+d\right)\left(v_{2}\right), \tilde{s}_{1}+d\right) & & \text { (by the contradiction hypothesis) } \\
& \leq T_{2}\left(\tilde{s}_{1}+d\right)\left(v_{2}\right), & & \text { (by definition in }(5))
\end{aligned}
$$

thus implying $d \leq 0$, a contradiction. This concludes the proof of

$$
T_{2}\left(\tilde{s}_{1}+d\right)\left(v_{2}\right)+d>T_{2}\left(\tilde{s}_{1}\right)\left(v_{2}\right)
$$

which we now use to show

$$
\left.O_{1}\left(\tilde{s}_{1}+d\right)\right)\left(v_{1}\right) \equiv T_{1}\left(T_{2}\left(\tilde{s}_{1}+d\right)\right)\left(v_{1}\right)<T_{1}\left(T_{2}\left(\tilde{s}_{1}\right)\right)\left(v_{1}\right)+d \equiv O_{1}\left(s_{1}\right)\left(v_{1}\right)+d
$$

and finish the proof of the lemma. Verification that $T_{1}$ is well-defined in the previous relation follows as for Lemma 1. Moreover, the previous relation is automatically true when $T_{1}\left(T_{2}\left(\tilde{s}_{1}+d\right)\right)\left(v_{1}\right)=0$. Therefore, by way of contradiction, suppose that for some $v_{1}$ we have $T_{1}\left(T_{2}\left(\tilde{s}_{1}+d\right)\right)\left(v_{1}\right) \geq T_{1}\left(T_{2}\left(\tilde{s}_{1}\right)\right)\left(v_{1}\right)+d$ and $T_{1}\left(T_{2}\left(\tilde{s}_{1}+d\right)\right)\left(v_{1}\right)>0$. We then have

$$
\begin{aligned}
T_{1}\left(T_{2}\left(\tilde{s}_{1}\right)\right)\left(v_{1}\right)+d & \leq T_{1}\left(T_{2}\left(\tilde{s}_{1}+d\right)\right)\left(v_{1}\right) & & \text { (by the contradiction hypothesis) } \\
& =v_{1}-r_{1}\left(T_{1}\left(T_{2}\left(\tilde{s}_{1}+d\right)\right)\left(v_{1}\right), T_{2}\left(\tilde{s}_{1}+d\right)\right) & & \left(\text { by } T_{1}\left(T_{2}\left(\tilde{s}_{1}+d\right)\right)\left(v_{1}\right)>0\right) \\
& =v_{1}-r_{1}\left(T_{1}\left(T_{2}\left(\tilde{s}_{1}+d\right)\right)\left(v_{1}\right)-d, d+T_{2}\left(\tilde{s}_{1}+d\right)\right) & & \left(\text { by definition of } r_{1}\right) \\
& \leq v_{1}-r_{1}\left(T_{1}\left(T_{2}\left(\tilde{s}_{1}\right)\right), d+T_{2}\left(\tilde{s}_{1}+d\right)\right) & & \text { (by the contradiction hypothesis) } \\
& \leq v_{1}-r_{1}\left(T_{1}\left(T_{2}\left(\tilde{s}_{1}\right)\right), T_{2}\left(\tilde{s}_{1}\right)\right) & & (\text { by }(33)) \\
& \leq T_{1}\left(T_{2}\left(\tilde{s}_{1}\right)\left(v_{1}\right),\right. & & \text { (by definition in }(5))
\end{aligned}
$$

thus implying $d \leq 0$, a contradiction. This concludes the proof of the lemma establishing discounting.

Proof of Lemma 3. Define $K^{N} \equiv \sum K_{i}$. The strategy of the proof follows the methodology in Cornes and Hartley (2007). We begin by showing a unique $K^{N}$ is consistent with system (15), that is the collection of 
"best responses" in (14), or

$$
K_{i}=\frac{1}{2} \int_{\underline{v}_{i}}^{\bar{v}_{i}} \max \left\{0, v_{i}-K^{N}+K_{i}\right\} f_{i}\left(v_{i}\right) d v_{i}
$$

Notice that $K^{N}=0$ cannot solve the system $(15)$, because $\bar{v}_{i}>0$. In what follows, we use $f_{i}\left(v_{i}\right)=0$ for $v_{i}<\underline{v}_{i}$ or $v_{i}>\bar{v}_{i}$. Define now $\sigma_{i} \equiv K_{i} / K^{N}$, so

$$
\begin{aligned}
\sigma_{i}=K_{i} / K^{N} & =\frac{1}{2} \mathrm{E}\left[\max \left\{0, v_{i}-K^{N}+K_{i}\right\}\right] \frac{1}{K^{N}} \quad \text { (by (14) and (15)) } \\
& =\frac{1}{2} \mathrm{E}\left[\max \left\{0, \frac{v_{i}}{K^{N}}-1+\sigma_{i}\right\}\right] \\
& =\frac{1}{2} \int_{K^{N}-K_{i}}^{\bar{v}_{i}}\left(\frac{v_{i}}{K^{N}}-1+\sigma_{i}\right) f_{i}\left(v_{i}\right) d v_{i} .
\end{aligned}
$$

Therefore, the relationship

$$
\sigma_{i}=\frac{1}{2} \int_{K^{N}-K_{i}}^{\bar{v}_{i}}\left(\frac{v_{i}}{K^{N}}-1+\sigma_{i}\right) f_{i}\left(v_{i}\right) d v_{i}
$$

implicitly describes $i$ 's expected share of expected contributions, in equilibrium. Note now that every solution of (15) must solve (35) with $\sum \sigma_{i}=1$. To show that at most one value of $K^{N}$ is consistent with these two requirements, we prove $\sigma_{i}$ is strictly decreasing in $K^{N}$ wherever $\sigma_{i}>0$. Differentiating both sides of (35) with respect to $K^{N}$, we obtain

$$
\begin{aligned}
\frac{d \sigma_{i}}{d K^{N}} & =-0+\frac{1}{2} \int_{K^{N}-K_{i}}^{\bar{v}_{i}}\left(-\frac{v_{i}}{\left(K^{N}\right)^{2}}+\frac{d \sigma_{i}}{d K^{N}}\right) f_{i}\left(v_{i}\right) d v_{i} \\
& =\frac{1}{2}\left(\frac{d \sigma_{i}}{d K^{N}} \int_{K^{N}-K_{i}}^{\bar{v}_{i}} f_{i}\left(v_{i}\right) d v_{i}-\frac{1}{\left(K^{N}\right)^{2}} \int_{K^{N}-K_{i}}^{\bar{v}_{i}} v_{i} f_{i}\left(v_{i}\right) d v_{i}\right) \\
& =\frac{d \sigma_{i}}{d K^{N}} \frac{1}{2}\left(1-F_{i}\left(K^{N}-K_{i}\right)\right)-\frac{1}{2\left(K^{N}\right)^{2}} \int_{K^{N}-K_{i}}^{\bar{v}_{i}} v_{i} f_{i}\left(v_{i}\right) d v_{i}
\end{aligned}
$$

and regrouping, we have

$$
\frac{d \sigma_{i}}{d K^{N}}\left(1+F_{i}\left(K^{N}-K_{i}\right)\right)=-\frac{1}{\left(K^{N}\right)^{2}} \int_{K^{N}-K_{i}}^{\bar{v}_{i}} v_{i} f_{i}\left(v_{i}\right) d v_{i}
$$

Now note that $K_{i}>0$, by (34), implies $K^{N}-K_{i}<\bar{v}_{i}$, so the right-hand side of the previous equation is strictly negative. We therefore conclude that if $K_{i}>0$, then $\sigma_{i}$ is strictly decreasing in $K^{N}$, as we wanted to show. Moreover, as (34) shows, if $K_{i}=0$ (and hence $\sigma_{i}=0$ ) for some value of $K^{N}, K_{i}$ remains equal to zero for all larger values of $K^{N}$. Therefore, only one $K^{N}$ is consistent with (35) and $\sum \sigma_{i}=1$ : as soon 
as we find one such value, and we keep increasing $K^{N}$, non-contributors remain non-contributors and $\sum \sigma_{i}$ strictly decreases away from 1 . Uniqueness of the equilibrium $K^{N}$ is sufficient to establish that at most one solution to the system of equations (15) exists, as at most one value of $K_{i}$ is consistent with each $K^{N}$. To see this, consider again equation (34) and rewrite it as

$$
K_{i}=\frac{1}{2} \int_{K^{N}-K_{i}}^{\bar{v}_{i}}\left(v_{i}-K^{N}+K_{i}\right) f_{i}\left(v_{i}\right) d v_{i} .
$$

The derivative of the right-hand side for $K_{i}$ is $\left(1-F_{i}\left(K^{N}-K_{i}\right)\right) / 2$, which is always smaller then the derivative of the left-hand side, which is 1 . Therefore, at most one value of $K_{i}$ can solve the previous equation.

Proof of Proposition 4. Totally differentiating (16) with respect to $K^{E}$ we obtain

$$
\left.0=-\frac{d K_{i}}{d K^{E}}-\frac{1}{2}\left[1-F_{i}\left(K^{E}+\sum_{j \neq i} K_{j}\right)\right)\right]\left[1+\sum_{j \neq i} \frac{d K_{j}}{d K^{E}}\right],
$$

which, for contributors, may be rewritten as

$$
-\frac{2}{1-F_{i}\left(K^{E}+\sum_{j \neq i} K_{j}\right)} \frac{d K_{i}}{d K^{E}}-\sum_{j \neq i} \frac{d K_{j}}{d K^{E}}=1 \quad \forall i
$$

while for non-contributors $F_{i}\left(K^{E}+\sum_{j \neq i} K_{j}\right)=1$ and $\frac{d K_{i}}{d K^{E}}=0$. Denote with $m \leq n$ the number of contributors in equilibrium. In what follows, all summations are taken over $m$. The system of equations (36) may be expressed in matrix form as

$$
\underbrace{\left(\begin{array}{ccccc}
\frac{2}{1-F_{1}\left(K^{E}+\sum_{j \neq 1} K_{j}\right)} & 1 & 1 & \ldots & 1 \\
1 & \frac{2}{1-F_{2}\left(K^{E}+\sum_{j \neq 2} K_{j}\right)} & 1 & \ldots & 1 \\
\ldots \ldots \ldots \ldots \ldots \ldots \ldots \ldots & \ldots \ldots & \ldots & \ldots \\
1 & 1 & \ldots & 1 & \frac{1}{1-F_{m}\left(K^{E}+\sum_{j \neq m} K_{j}\right)}
\end{array}\right)}_{\text {A }} \underbrace{\left(\begin{array}{c}
-d K_{1} / d K^{E} \\
-d K_{2} / d K^{E} \\
\vdots \\
-d K_{m-1} / d K^{E} \\
-d K_{m} / d K^{E}
\end{array}\right)}_{-\Delta}=\underbrace{\left(\begin{array}{c}
1 \\
1 \\
1 \\
1 \\
1
\end{array}\right)}_{1}
$$

The $m \times m$ matrix $A$ may be shown to be non-singular, so the implicit function theorem is applicable. To establish part a, we use Farkas' Lemma: $-\Delta \geq \mathbf{0}$ if there is no solution $\mathbf{y}$ to $\mathrm{A}^{T} \mathbf{y} \geq \mathbf{0}$ with $\mathbf{1}^{T} \mathbf{y}<0$. By contradiction, assume such a vector $\mathbf{y}$ exists, and consider the first element of $\mathrm{A}^{T} \mathbf{y}$ :

$$
\left(\frac{2}{1-F_{1}\left(\sum_{j \neq 1} K_{j}+K^{E}\right)}\right) y_{1}+y_{2}+\ldots+y_{m} .
$$


From $\mathbf{1}^{T} \mathbf{y}<0$, we have $y_{2}+\ldots+y_{m}<-y_{1}$, so the expression in (38) is strictly smaller than

$$
\left[\frac{2}{1-F_{1}\left(\sum_{j \neq 1} K_{j}+K^{E}\right)}-1\right] y_{1} .
$$

Because the term in square brackets is always positive, the sign of the previous expression only depends on $y_{1}$. In particular, if $y_{1}<0$, the chain of inequalities above yields that the term in (38) is strictly negative, a contradiction to $\mathrm{A}^{T} \mathbf{y} \geq \mathbf{0}$. Therefore, we must have $y_{1} \geq 0$. Repeating this reasoning for all elements of $\mathrm{A}^{T} \mathbf{y}$ we obtain that all elements of $\mathbf{y}$ are non-negative, thus contradicting $\mathbf{1}^{T} \mathbf{y}<0$. Farkas' Lemma then implies $(-\Delta) \geq \mathbf{0}$, or that $d K_{i} / d K^{E} \leq 0$ for all $i$; furthermore, at least one inequality must hold strictly since otherwise the right-hand side of (37) would be $\mathbf{0}$. It only remains to show that $d K_{i} / d K^{E}<0$ for all $i$. For simplicity, we only consider the case where $d K_{1} / d K^{E}=0$ and $d K_{2} / d K^{E}<0$ and show this leads to a contradiction. Then the first element of the product $\mathrm{A}(-\Delta)$ is

$$
-\frac{d K_{2}}{d K^{E}}-\frac{d K_{3}}{d K^{E}}-\cdots-\frac{d K_{m}}{d K^{E}}
$$

which is strictly greater than the second element,

$$
-\left(\frac{2}{1-F_{2}\left(\sum_{j \neq 2} K_{j}+K^{E}\right)}\right) \frac{d K_{2}}{d K^{E}}-\frac{d K_{3}}{d K^{E}}-\frac{d K_{m}}{d K^{E}}
$$

Therefore, (39) and (40) cannot both equal 1, contradicting (37). This completes the proof of part a.

To establish part b, we sum all equations in (37) and obtain

$$
-m=\sum\left[(m-1)+\frac{2}{1-F_{i}\left(\sum_{j \neq i} K_{j}+K^{E}\right)}\right] \frac{d K_{i}}{d K^{E}}<(m+1) \sum \frac{d K_{i}}{d K^{E}},
$$

where the inequality follows from part a and the fact that the terms in square brackets is always larger than $(m+1)$. Thus, we obtain $\sum d K_{i} / d K^{E}>-m /(m+1) \geq-n /(n+1)$, establishing part b. 


\section{References}

Admati, Anat R., and Motty Perry (1991), Joint projects without commitment, Review of Economic Studies $58,259-276$.

Alboth, Dirk, Anat Lerner, and Jonathan Shalev (2001), Profit maximizing in auctions of public goods, Journal of Public Economic Theory 3, 501-525.

Alesina, Alberto, and Eliana La Ferrara (2000), Participation in heterogeneous communities, Quarterly Journal of Economics 115, 847-904.

Alesina, Alberto, and Eliana La Ferrara (2005), Ethnic diversity and economic performance, Journal of Economic Literature 43, 847-904.

Athey, Susan (2001), Single crossing properties and the existence of pure strategy equilibria in games of incomplete information, Econometrica 69, 861-889.

Bagnoli, Mark, and Barton L. Lipman (1989), Provision of public goods: fully implementing the core through private contributions, Review of Economic Studies 56, 583-601.

Barbieri, Stefano, and David A. Malueg (2008), Private provision of a discrete public good: continuousstrategy equilibria in the private-information subscription game, Journal of Public Economic Theory $10,529-545$.

Cadsby, Charles B., and Elizabeth Maynes (1999), Voluntary provision of threshold public goods with continuous contributions: experimental evidence, Journal of Public Economics 71, 53-73.

Cornes, Richard, and Roger Hartley (2007), Aggregative public good games, Journal of Public Economic Theory 9, 201-219.

Greif, A., (1993), Contract enforceability and economic institutions in early trade: the Maghribi traders' coalition, American Economic Review 83, 525-48.

Holmström, Bengt, and Roger B. Myerson (1983), Efficient and durable decision rules with incomplete information, Econometrica 51, 1799-1820.

Jackman, Mary R., and Marie Crane (1986), Some of my best friends are black..., The Public Opinion Quarterly, 50, 459-486.

Laussel, Didier, and Thomas R. Palfrey (2003), Efficient equilibria in the voluntary contributions mechanism with private information, Journal of Public Economic Theory 5, 449-478. 
Ledyard, John O., and Thomas R. Palfrey (1999), A characterization of interim efficiency with public goods, Econometrica $67,435-448$.

Ledyard, John O., and Thomas R. Palfrey (2007), A general characterization of interim efficient mechanisms for independent linear environments, Journal of Economic Theory 133, 441-466.

Mailath, George, and Andrew Postlewaite (1990), Asymmetric information bargaining problems with many agents, The Review of Economic Studies 57, 351-367.

Marks, Melanie B., and Rachel T. A. Croson (1999), The effect of incomplete information in a threshold public goods experiment, Public Choice 99, 103-118.

Martimort, David, and Humberto Moreira (2007), Common agency with informed principals, mimeo.

McBride, Michael (2006), Discrete public goods under threshold uncertainty, Journal of Public Economics $90,1181-1199$.

Menezes, Flavio M., Paulo K. Monteiro, and Akram Temimi (2001), Private provision of discrete public goods with incomplete information, Journal of Mathematical Economics 35, 493-514.

Nitzan, Shmuel, and Richard E. Romano (1990), Private provision of a discrete public good with uncertain cost, Journal of Public Economics 42, 357-370.

Potters, Jan, and Randolph Sloof (1996), Interest groups: a survey of empirical models that try to assess their influence, European Journal of Political Economy 12, 403-442.

Palfrey, Thomas R., and Howard Rosenthal (1984), Participation and the provision of discrete public goods: a strategic analysis, Journal of Public Economics 24, 171-193.

Satterthwaite, Mark A., and Steven R. Williams (1989), Bilateral trade with the sealed bid k-double auction: existence and efficiency, Journal of Economic Theory 48, 107-133. 